\title{
Compositional study of asteroids in the Erigone collisional family using visible spectroscopy at the $10.4 \mathrm{~m} \mathrm{GTC}$
}

\author{
David Morate ${ }^{1,2}$, Julia de León ${ }^{1,2}$, Mário De Prá ${ }^{3}$, Javier Licandro ${ }^{1,2}$, Antonio Cabrera-Lavers ${ }^{1,4}$, \\ Humberto Campins ${ }^{5}$, Noemí Pinilla-Alonso ${ }^{6}$, and Víctor Alí-Lagoa ${ }^{7}$ \\ ${ }^{1}$ Instituto de Astrofísica de Canarias (IAC), C/vía Láctea s/n, 38205 La Laguna, Tenerife, Spain \\ e-mail: damog@iac.es \\ 2 Departamento de Astrofísica, Universidad de La Laguna, 38205 La Laguna, Tenerife, Spain \\ 3 Observatório Nacional, Coordenação de Astronomia e Astrofísica, 20921-400 Rio de Janeiro, Brazil \\ 4 GTC Project Office, 38205 La Laguna, Tenerife, Spain \\ 5 Physics Department, University of Central Florida, PO Box 162385, Orlando, FL 32816-2385, USA \\ 6 Department of Earth and Planetary Sciences, University of Tennessee, Knoxville, 37996 TN, USA \\ 7 Laboratoire Lagrange, OCA, Boulevard de l'Observatoire, BP 422906304 Nice Cedex 04, France
}

Received 25 September 2015 / Accepted 10 December 2015

\begin{abstract}
Two primitive near-Earth asteroids, (101955) Bennu and (162173) Ryugu, will be visited by a spacecraft with the aim of returning samples back to Earth. Since these objects are believed to originate in the inner main belt primitive collisional families (Erigone, Polana, Clarissa, and Sulamitis) or in the background of asteroids outside these families, the characterization of these primitive populations will enhance the scientific return of the missions. The main goal of this work is to shed light on the composition of the Erigone collisional family by means of visible spectroscopy. Asteroid (163) Erigone has been classified as a primitive object, and we expect the members of this family to be consistent with the spectral type of the parent body. We have obtained visible spectra $(0.5-0.9 \mu \mathrm{m})$ for 101 members of the Erigone family, using the OSIRIS instrument at the $10.4 \mathrm{~m}$ Gran Telescopio Canarias. We found that $87 \%$ of the objects have typically primitive visible spectra consistent with that of (163) Erigone. In addition, we found that a significant fraction of these objects $(\sim 50 \%)$ present evidence of aqueous alteration.
\end{abstract}

Key words. minor planets, asteroids: general - methods: data analysis - techniques: spectroscopic

\section{Introduction}

Primitive asteroids are considered to be composed of the most pristine materials in the Solar System, being remnants of the processes that followed the condensation of the protoplanetary nebula and the formation of the planets. The materials on these objects have been altered over time by different processes, such as space weathering and aqueous alteration (Fornasier et al. 2014). Aqueous alteration mainly acts on primitive asteroids $(\mathrm{C}, \mathrm{B}$, and low-albedo X-types, according to the DeMeo et al. (2009) classification scheme), producing a low-temperature $(<320 \mathrm{~K})$ chemical alteration of the materials that is due to the presence of liquid water. This water acts as a solvent and generates hydrated materials such as phyllosilicates, sulfates, oxides, carbonates, and hydroxides. The presence of hydrated materials thus implies that liquid water was present in the primordial asteroids, produced by the melting of water ice by a heating source (Fornasier et al. 2014). The most unambiguous indicator of hydration is the $3 \mu \mathrm{m}$ hydration band observed in infrared photometry and spectroscopy of many primitive asteroids. This feature is correlated with the $0.7 \mu \mathrm{m} \mathrm{Fe}{ }^{2+} \rightarrow \mathrm{Fe}^{+3}$ oxidized iron absorption band observed in the visible spectra of these asteroids (Vilas 1994; Howell et al. 2011; Rivkin 2012).

Except for this aqueous alteration, primitive asteroids have undergone minimal geological or thermal evolution, experiencing, on the contrary, an intense collisional evolution that affected their shape, size, and surface composition. Therefore, studying the products of these collisional events and their mineralogical compositions will shed light on the evolutionary history of the Solar System.

Asteroid collisional families are groups of asteroids sharing very similar orbital properties (Hirayama 1918) that are thought to be the direct result of energetic collisional events. Spectroscopic observations provided the first confirmation of the collisional origin of a family: the Vesta family (Binzel \& Xu 1993). The number of spectroscopic studies of collisional families has steadily increased since then, focusing on the characterization of their mineralogy. Additionally, considering that nearEarth asteroids (NEAs) come primarily from the main asteroid belt (Bottke et al. 2002), collisional families are good sources of NEAs, as they generate plenty of small fragments during their formation. Families located close to particular resonances in the belt can easily send these fragments to the near-Earth space. In this sense, the inner asteroid belt (the region located between the $v_{6}$ resonance, near 2.15 AU, and the 3:1 mean-motion resonance with Jupiter, at $2.5 \mathrm{AU}$ ) is considered as a primary source of NEAs (Bottke et al. 2002).

The NASA OSIRIS-REx (Lauretta et al. 2010) and JAXA ${ }^{1}$ Hayabusa 2 (Tsuda et al. 2013) sample-return missions have targeted two NEAs: (101955) Bennu and (162173) Ryugu,

\footnotetext{
Japan Aerospace Exploration Agency.
} 
respectively. These are primitive asteroids that are believed to originate in the inner belt, where five distinct sources have been identified: four primitive collisional families (Polana, Erigone, Sulamitis, and Clarissa) and a population of low-albedo and lowinclination background asteroids (Campins et al. 2010, 2013; Bottke et al. 2015). Identifying and characterizing the populations from which these two NEAs might originate will enhance the science return of both missions.

With this main objective in mind, we initiated a spectroscopic survey in the visible and the near-infrared in 2010 to characterize the primitive collisional families in the inner belt and the low-albedo background population (PRIMitive Asteroid Spectroscopic Survey, PRIMASS). We started with the largest one, the Polana family (Pinilla-Alonso et al. 2016; de León et al. 2016), using, among others, visible spectra obtained with the $10.4 \mathrm{~m}$ Gran Telescopio Canarias, which is located at the El Roque de los Muchachos Observatory on the island of La Palma (Spain). We found that despite the dynamical and collisional complexity of the Polana family (Walsh et al. 2013; Milani et al. 2014; Dykhuis \& Greenberg 2015), there is a spectral homogeneity both in the visible and near-infrared wavelengths: all the asteroids show a continuum in spectral slopes from blue to moderately red that is typical of B- and C-type primitive asteroids.

To continue with the PRIMASS survey, we have observed and characterized the Erigone family, the second largest of the four primitive collisional families in the inner belt. We have performed our analysis using data obtained with the $10.4 \mathrm{~m}$ Gran Telescopio Canarias during the semester 2014B (September 2014-February 2015). In Sect. 2 we describe the observations and the data reduction. In Sect. 3 we present the analysis performed on the data, including taxonomical classification, computation of spectral slopes, and analysis of aqueous alteration. In Sect. 4 we discuss the obtained results, and in Sect. 5 we summarize the conclusions.

\section{Observations and data reduction}

The sample of asteroids we observed in this study has been selected using the Minor Planet Physical Properties Catalogue $^{2}\left(\mathrm{MP}^{3} \mathrm{C}\right)$, which acknowledges the NASA Planetary Data System (PDS) as a data source. Orbital data of the asteroid families are extracted from a dataset containing asteroid dynamical families including both analytic and synthetic proper elements. These families were computed by David Nesvorny (Nesvorny 2012) using his code based on the hierarchical clustering method (HCM), as described in Zappala et al. (1990) and Zappala \& Cellino (1994). The $\mathrm{MP}^{3} \mathrm{C}$ catalog also provides information on the absolute magnitude $H$, the diameter $D$, and the geometric albedo $p_{V}$. For the diameters and albedos we used the values provided by WISE (Wide-field Infrared Survey Explorer, Masiero et al. 2011). Family membership is based on values of the synthetic proper elements, that is, on the semimajor axis $(a)$, eccentricity $(e)$ and inclination $(i)$, and also on the absolute magnitude as a function of semimajor axis $(a, H)$. According to these parameters, the Erigone family contains a total of 1785 asteroids.

The selection criterion was quite simple. The Erigone collisional family is a primitive one according to the information we currently have: from the list of 1785 members of the family, 1015 have no albedo information, but from the remaining 770 objects, 692 have geometric albedo values $p_{V}<0.1$. In addition, 156 objects have SDSS color-based taxonomies, the majority of

\footnotetext{
2 http://mp3c.oca.eu/MP3C/
}

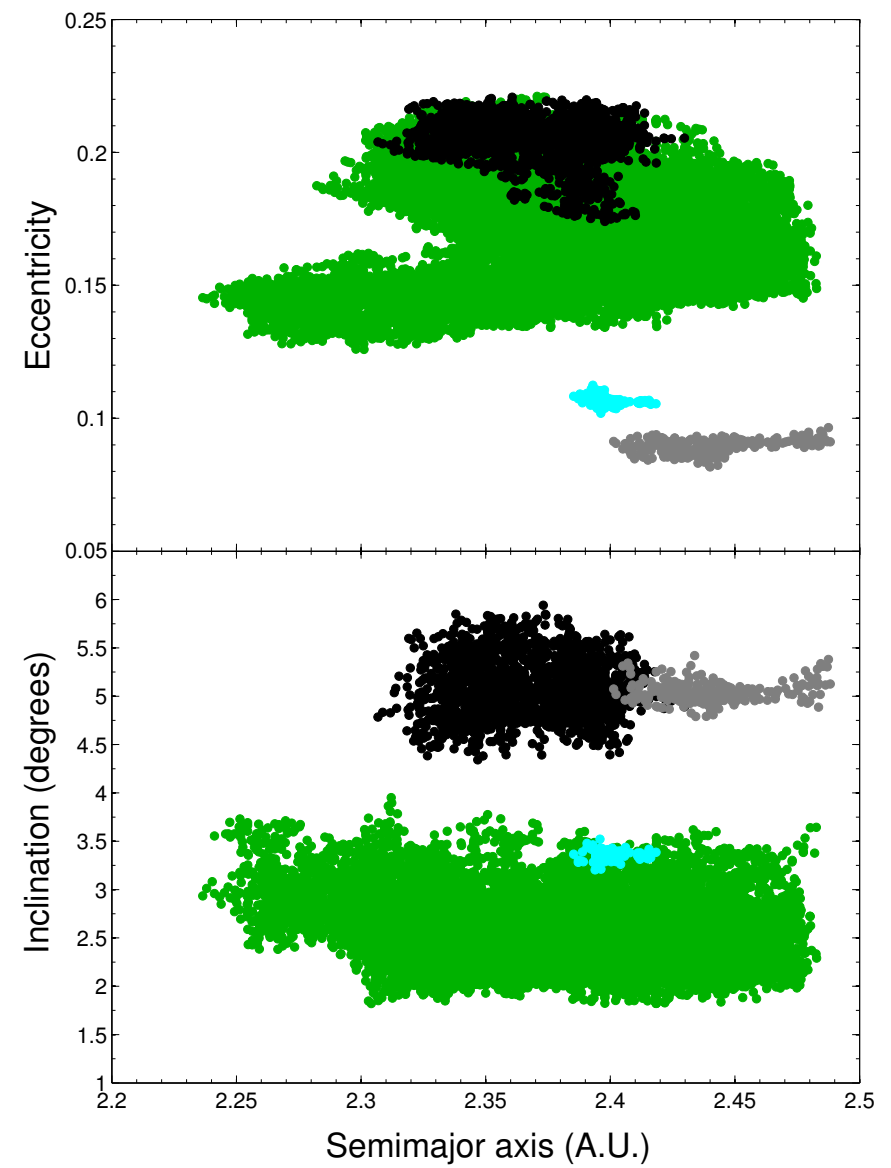

Fig. 1. Proper semimajor axis $(a)$ versus proper eccentricity $(e)$ and proper inclination $(i)$ for the primitive asteroid families in the inner main belt. The Erigone family is depicted in black. The Polana (Nysa-Polana complex in this plot), Sulamitis, and Clarissa families are depicted in green, gray, and cyan, respectively.

them belonging to primitive classes (91 C-types, 10 B-types, 7 X-types, 39 S-types, 5 L-types, 2 K-types, 1 V-type, and 1 A-type). Therefore, we selected those asteroids with geometric albedo $p_{V}<0.1$. Observations were made in service mode on different nights (see details in the next section). From the previous list of asteroids, we selected those with an apparent visual magnitude in the range $18<m_{V}<21$ for a good compromise between the number of asteroids observed and the signalto-noise ratios of the spectra. This means that for some of the nights there were no visible asteroids fulfilling these criteria. In these cases (16 in total), we first tried to find objects with an SDSS color-based taxonomical classification available (preferentially C-types). When this information was not available, we selected objects with $p_{V}>0.1$. Our last option was to select objects without information on their albedo. We describe this small subsample in detail in Sect. 3.

\subsection{Observations}

We obtained low-to-intermediate resolution visible spectroscopy for a total of 101 asteroids using the Optical System for Imaging and Low Resolution Integrated Spectroscopy (OSIRIS) camera spectrograph (Cepa et al. 2000; Cepa 2010) at the $10.4 \mathrm{~m}$ Gran Telescopio Canarias (GTC), located at the El Roque de los Muchachos Observatory (ORM) in La Palma, Canary Islands, Spain. The OSIRIS instrument consists of a mosaic of two 
Marconi CCD detectors, each with $2048 \times 4096$ pixels and a total unvignetted field of view of $7.8 \times 7.8 \mathrm{arcmin}$. The single pixel physical size is $15 \mu \mathrm{m}$, giving a plate scale of $0.127^{\prime \prime} /$ pix. To increase the signal-to-noise ratio for our observations, we selected the $2 \times 2$ binning mode with a readout speed of $200 \mathrm{kHz}$ (which has a gain of $0.95 \mathrm{e}-/ \mathrm{ADU}$ and a readout noise of $4.5 \mathrm{e}-$ ), which corresponds to the standard operation mode of the instrument.

All the spectra were obtained using the OSIRIS R300R grism, which produces a dispersion of $7.74 \AA$ 解 for a $0.6^{\prime \prime}$ slit (in the worst-case scenario, a seeing of 3.0" would translate into a resolution of $38.7 \AA /$ pix), with a spectral coverage from 4800 to $10000 \AA$. The R300R grism is used in combination with a second-order spectral filter. However, the spectrum is still slightly contaminated, with a distinguishable contribution for wavelengths at 4800-4900 $\AA$ and 9600-9800 $\AA$. To be conservative, we therefore did not consider data beyond $9000 \AA$ here. A 5.0" slit was used to account for possible variable seeing conditions, and it was oriented to the parallactic angle to minimize loses due to atmospheric dispersion. Series of three spectra (whenever possible) were taken for all the targets, with exposure times ranging from 150-600 s, depending on the target brightness. Observational details are listed in Table C.1. Information includes asteroid number, date of observation, starting UT, airmass, exposure time, solar analog stars, and seeing at the moment of the observations. Consecutive spectra were shifted in the slit direction by 10 arcsecs to improve the sky subtraction and fringing correction.

Observations were made in service mode (within GTC programs GTC39-14B and GTC18-15A) on different nights from September ${ }^{3} 2014$ to May 2015. Night conditions were very variable, covering a wide range of different weather conditions. This was because the program was classified as a "filler" ( $C$ band) program within the GTC nightly operation schedule. The aim of this type of program is to obtain spectra with a high signalto-noise ratio for targets that are relatively bright for a $10 \mathrm{~m}$ class telescope such as GTC in non-optimal weather conditions, which would include a high seeing value (higher than 1.5 arcsec), bright moon, or some cirrus coverage. Because of this, spectra quality might vary from one night to another (see Table C.1, last column). Since the weather conditions (i.e., clouds, sky brightness, etc.) are the main constraint during the observation, this variation is unrelated to the target brightness. For completeness, we included in this study the visible spectra of asteroids (163) Erigone, the parent body of the family, and (571) Dulcinea, the second largest asteroid in the family, both from the SMASS II catalog ${ }^{4}$. There were no other asteroids from the Erigone family with published visible spectra. All in all, our sample of asteroids from the Erigone collisional family includes a total of 103 objects.

\subsection{Data reduction}

A reduction pipeline for asteroid spectroscopic data obtained with the GTC was developed to optimize the reduction process. This pipeline combines standard IRAF $^{5}$ tasks and some MATLAB functions.

\footnotetext{
3 Some of the asteroids were observed during August 2014 upon request from the GTC operations team to fill observational gaps during particular nights.

4 Available at http://smass.mit.edu/catalog.php

5 IRAF is distributed by the National Optical Astronomy Observatories, which are operated by the Association of Universities for Research in Astronomy, Inc., under cooperative agreement with the National Science Foundation.
}

Table 1. Equatorial coordinates of the solar analog stars used to obtain the reflectance spectra of the observed asteroids.

\begin{tabular}{llcc}
\hline \hline ID & Star & $\alpha$ & $\delta$ \\
\hline 1 & SA 93-101 & $01: 53: 18.0$ & $+00: 22: 25$ \\
2 & SA 98-978 & $06: 51: 34.0$ & $-00: 11: 28$ \\
3 & SA 102-1081 & $10: 57: 04.4$ & $-00: 13: 10$ \\
4 & SA 110-361 & $18: 42: 45.0$ & $+00: 08: 04$ \\
5 & SA 112-1333 & $20: 43: 11.8$ & $+00: 26: 15$ \\
6 & SA 115-271 & $23: 42: 41.8$ & $+00: 45: 10$ \\
7 & SA 107-998 & $15: 38: 16.4$ & $+00: 15: 23$ \\
\hline
\end{tabular}

With the IRAF tasks included in our pipeline, images were initially bias- and flat-field corrected using lamp flats from the GTC instrument calibration module. Sky background was then subtracted, and a one-dimensional spectrum was extracted with an extraction aperture that varied depending on the seeing of the corresponding night. After the extraction, the one-dimensional spectra were wavelength calibrated with $\mathrm{Xe}+\mathrm{Ne}+\mathrm{HgAr}$ lamps. As a final step, the three spectra of the same object, when available, were averaged to obtain one final spectrum of the asteroid.

To correct for telluric absorptions and obtain relative reflectance spectra, at least one solar analog star from the Landolt catalog (Landolt 1992) was observed each night. When possible, more than one solar analog star was observed to improve the quality of the final spectra and to minimize potential variations in spectral slope introduced by the use of one single star. These stars were observed using the same spectral configuration as for the asteroids, and at a similar airmass. The list of the solar analogs used in this study is shown in Table 1.

The MATLAB routines in our pipeline were used to align the spectra of the objects and the corresponding solar analog with the theoretical wavelength positions of the telluric lines at 6867.19 and $7593.70 \AA$. After they were aligned, the spectrum of the object was divided by that of the solar analog, and the result was normalized to unity at $0.55 \mu \mathrm{m}$. When more than one solar analog was observed, we divided the spectrum of the asteroid by the spectra of the stars and checked against any possible variations in spectral slopes, which were on the order of $0.6 \% / 1000 \AA$. A variation smaller than $1 \% / 1000 \AA$ is typically considered as a good value.

After we obtained the final spectrum for each object, we applied a binning to each spectrum, taking intervals of 11 points as the bin size. Then, the reflectance value corresponding to the central wavelength of the bin size was substituted with the median reflectance value to avoid spectral disturbances, and thus making the resulting spectrum more robust. To choose the binning size, we selected the worst spectrum in our sample and applied different binning sizes until its quality improved. Since the finest spectral feature we wished to measure, the $0.7 \mu \mathrm{m}$ band, has an approximate width of $2000 \AA$, we consider that the selected binning is sufficient to improve the quality of the spectra and to not affect the obtained results. The spectral range extends from 0.5 to $0.9 \mu \mathrm{m}$, with a step of $0.0055 \mu \mathrm{m}$. Spectra are shown in Figs. A.1 and B.1.

\section{Analysis and results}

After computing the final spectra, a taxonomic classification was made using M4AST ${ }^{6}$, which is an online tool for modeling asteroid spectra (Popescu et al. 2012). The method used by the

6 http://m4ast.imcce.fr/ 

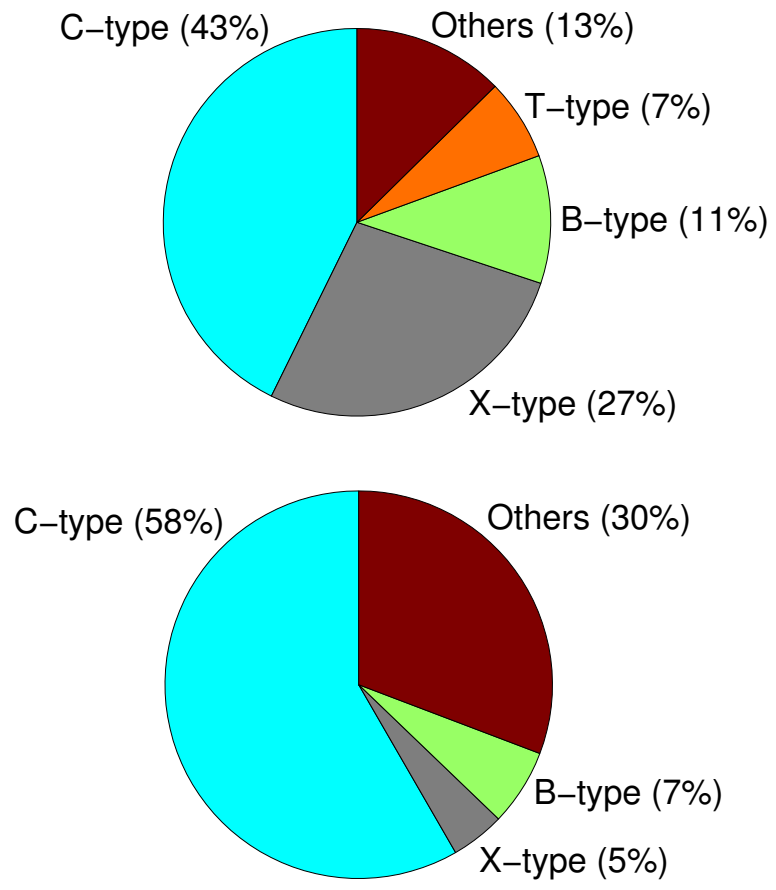

Fig. 2. Distribution of the taxonomical classes for the Erigone family. The top panel shows the distribution for the sample of 103 asteroids we studied here. The bottom panel shows the distribution for the colorbased taxonomy from the SDSS data (the X class in the SDSS classification includes the T-type from the Bus taxonomy). The "Others" class includes all non-primitive taxonomies (S-types, V-types, and L-types).

M4AST tool to classify asteroid spectra is the following: first, the spectrum is fitted with a polynomial curve, and then this curve is compared to each of the classes defined by the DeMeo et al. (2009) taxonomy at the corresponding wavelengths. The tool then selects the taxonomical class producing the smallest standard deviation.

We worked with spectra in the visible wavelength range and therefore applied the Bus \& Binzel (2002) taxonomy, in which most of the classes overlap with those of DeMeo et al. (2009). We individually checked those cases in which the taxonomical classes were exclusive to the DeMeo et al. (2009) taxonomy and visually classified them according to Bus \& Binzel (2002).

To obtain robust results, both original and binned spectra were classified using the M4AST tool. A $\chi^{2}$ method (Bevington $\&$ Robinson 1992) was used to test how well the spectra fit the templates. The chosen result corresponded to the smallest standard deviation. Whenever a spectrum was very different from the best-fitting template, or when the standard deviations were very similar for two or more taxonomical classes, we simplified the method: the binned spectrum was fit to a third-order polynomial, and then the procedure was repeated. The taxonomical classification obtained for each asteroid is shown in the last column of Table C.2.

The classification yielded a total of $44 \mathrm{C}$-type asteroids (including the subclasses $\mathrm{Cb}, \mathrm{Cg}, \mathrm{Ch}$, and $\mathrm{Cgh}$ ), $28 \mathrm{X}$-type asteroids (and subclasses Xc and Xk), 11 B-types, 7 T-types, and 13 objects with non-primitive classifications: 6 S-types (and subclasses), 1 V-type, and 6 L-types. These results are illustrated with a pie chart in Fig. 2 (upper panel). As expected from our selection criterion (objects with $p_{V}<0.1$ ), the majority of the asteroids belong to primitive taxonomical classes (C-, B-, X-, and T-). As mentioned in Sect. 2 and because of observational
Table 2. Asteroids in our sample without information on their visible geometric albedo or whose albedo value is higher than $10 \%\left(p_{V}>0.1\right)$.

\begin{tabular}{lccc}
\hline \hline Asteroid & $p_{v}$ & SDSS Class. & M4AST Class. \\
\hline 38661 & - & $\mathrm{S}$ & $\mathrm{Sr}$ \\
39895 & - & $\mathrm{S}$ & $\mathrm{S}$ \\
56349 & - & $\mathrm{C}$ & $\mathrm{B}$ \\
186446 & - & $\mathrm{C}$ & $\mathrm{Ch}$ \\
\hline 18759 & 0.303 & - & $\mathrm{Sr}$ \\
24037 & 0.129 & - & $\mathrm{L}$ \\
132383 & 0.356 & - & $\mathrm{L}$ \\
\hline 38106 & - & - & $\mathrm{L}$ \\
50068 & - & - & $\mathrm{L}$ \\
66403 & - & - & $\mathrm{Ch}$ \\
69706 & - & - & $\mathrm{S}$ \\
70511 & - & - & $\mathrm{V}$ \\
76922 & - & - & $\mathrm{Xk}$ \\
85727 & - & - & $\mathrm{S}$ \\
107070 & - & - & $\mathrm{L}$ \\
166264 & - & - & $\mathrm{Xk}$ \\
186446 & - & - & $\mathrm{Ch}$ \\
\hline
\end{tabular}

constraints, 16 asteroids from the 101 observed did not fulfill the selection criteria. Their taxonomical classification is shown in Table 2. Moreover, the three asteroids with $p_{V}>0.1$ correspond to non-primitive classes. The remaining ten asteroids without albedo information show a mixture of taxonomies, with two C-types, two X-types, two S-types, three L-types, and one V-type. From the total of 86 asteroids with $p_{V}<0.1$, only one single object has a non-primitive classification, which is an L-type.

To perform one final comparison with the taxonomical distribution we found from our visible spectra, we searched for all the asteroids in the Erigone family with an SDSS color-based taxonomy. A total of 156 objects belonging to the Erigone family were classified according to (DeMeo \& Carry 2013). In the lower panel of Fig. 2 we show their taxonomic distribution. The proportion of C-and B-types from our sample and the one from the SDSS taxonomy agree well: the proportion of X-type asteroids is significantly larger in our case. The difference between the two non-primitive distributions is probably due to the selection criterion $\left(p_{V}<0.1\right)$.

Figure 3 shows the distribution in the $(a, H)$ space of the 1785 asteroids that have been identified as members of the Erigone collisional family (gray circles). As described by Vokrouhlický et al. (2006), the family shows signs of having experienced dynamical spreading through Yarkovsky thermal forces. Solid curves in Fig. 3 define the boundaries of the family, also known as the Yarkovsky cone, which is computed using the following expression:

$0.2 H=\log _{10}(\Delta a / C)$,

where $\Delta a=a-a_{\mathrm{c}}$, with $a_{\mathrm{c}}$ defined as the center of the family. In practice, $a_{\mathrm{c}}$ is often close to, or the same as, the semimajor axis of the largest member of the family, in this case, asteroid (163) Erigone (Vokrouhlický et al. 2006; Bottke et al. 2015). Bottke et al. (2015) showed that for the Erigone family, $C=1.9 \times 10^{-5}$. This Yarkovsky cone is basically an envelope around the center of the family, indicating the farthest distance that a family member can drift as a function of its size. Objects outside this cone are most likely family interlopers. Figure 3 shows the position, with respect to this cone, of the asteroids studied in this work. Different colors are associated with different spectral classes, as indicated in the legend of the figure. It is interesting to note that 


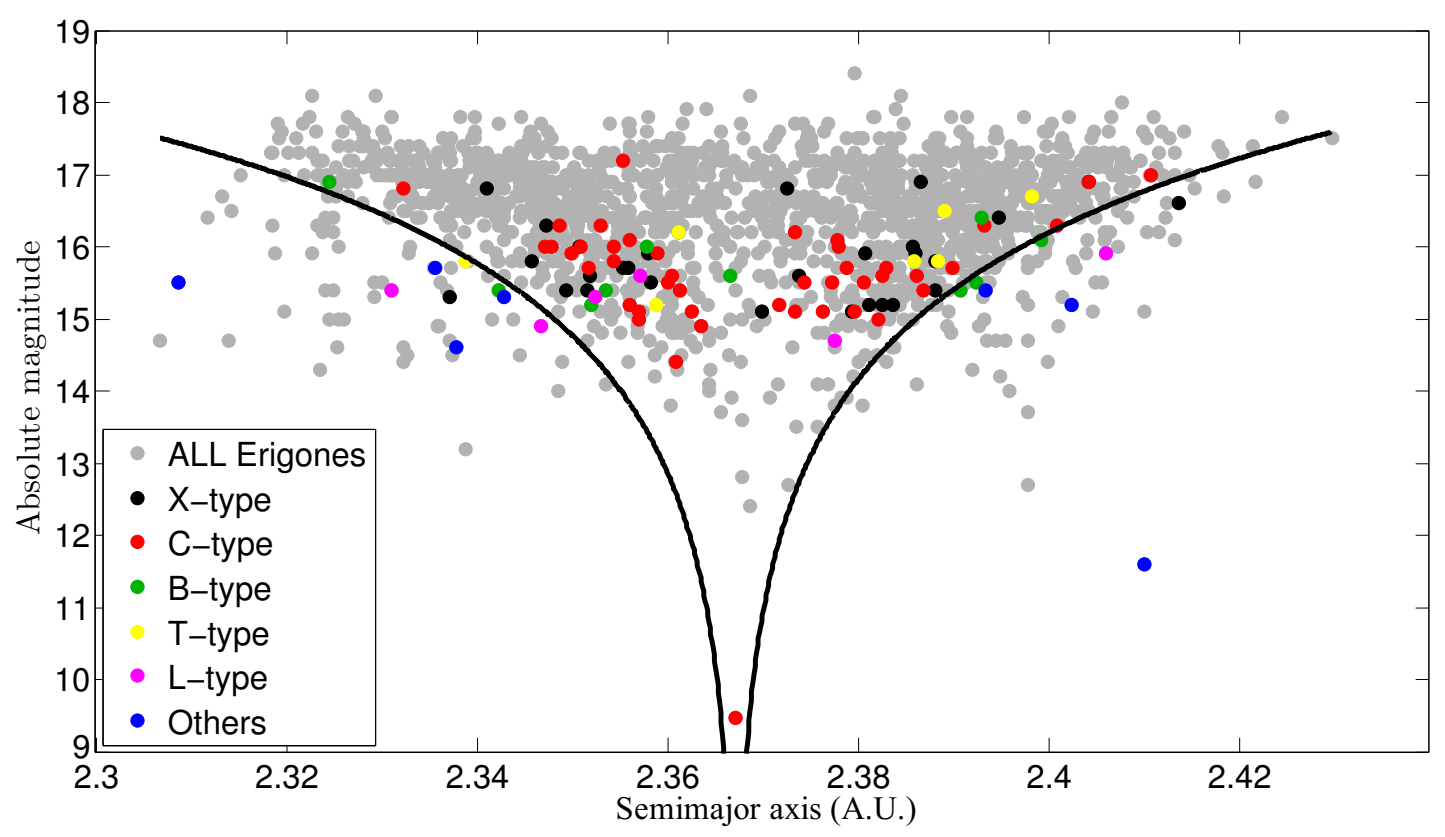

Fig. 3. Absolute magnitude $(H)$ of the asteroids from the Erigone family as a function of their proper semimajor axis. The Erigone family (a total of 1785 objects) is shown in gray. The colored circles correspond to the different taxonomical classes found for our sample of 103 members: C-types (red), X-types (black), B-types (green), T-types (yellow), and the remaining non-primitive classes (S-types, L-types, and V-types, in blue). The solid lines represent the boundaries of the family (so-called Yarkovsky cone). The object with the lowest value of $H$ that is located at the bottom of this cone is the parent body of the family, asteroid (163) Erigone.

most of the asteroids with non-primitive taxonomies fall outside the family boundaries, confirming that they are most likely interlopers.

In the following sections we perform a more detailed analysis of the asteroids of the Erigone family with a primitive taxonomical classification, that is, C-, X-, B-, and T-types. A total of 90 objects have been analyzed.

\subsection{Spectral slopes}

Given that primitive asteroids have featureless, linear spectra, we started by computing the spectral slope $S^{\prime}$, as defined by (Luu \& Jewitt 1990), between 0.55 and $0.90 \mu \mathrm{m}$ :

$S^{\prime}=\left(\frac{\mathrm{d} S / \mathrm{d} \lambda}{S_{0.55}}\right)$,

where $\mathrm{d} S / \mathrm{d} \lambda$ is the rate of change of the reflectivity in the aforementioned wavelength range, and $S_{0.55}$ is the reflectivity at 0.55 microns. To compute it, a linear least-squares fit between 0.55 and $0.90 \mu \mathrm{m}$ was applied to every primitive asteroid spectra. We normalized ${ }^{7}$ the slope at $0.55 \mu \mathrm{m} . S^{\prime}$ is measured in units of $\% / 1000 \AA$. The resulting values for the computed spectral slopes are shown in Table C.3. The slope errors take into account both the $1 \sigma$ uncertainty of the linear fit and the variation of $0.6 \% / 1000 \AA$, which is attributable to the use of different solar analog stars during the night (see Sect. 2.2 for more details). Figure 4 shows the distribution of the computed slopes for the 90 primitive objects of the Erigone collisional family (red). As a comparison we show the distribution of the visible spectra slopes of the asteroids of the Polana family (blue) from de León et al. (2016), which are compatible with a B-type parent. The

\footnotetext{
7 This is the central wavelength of the Johnson $V$ filter, which is usually used as normalization reference.
}

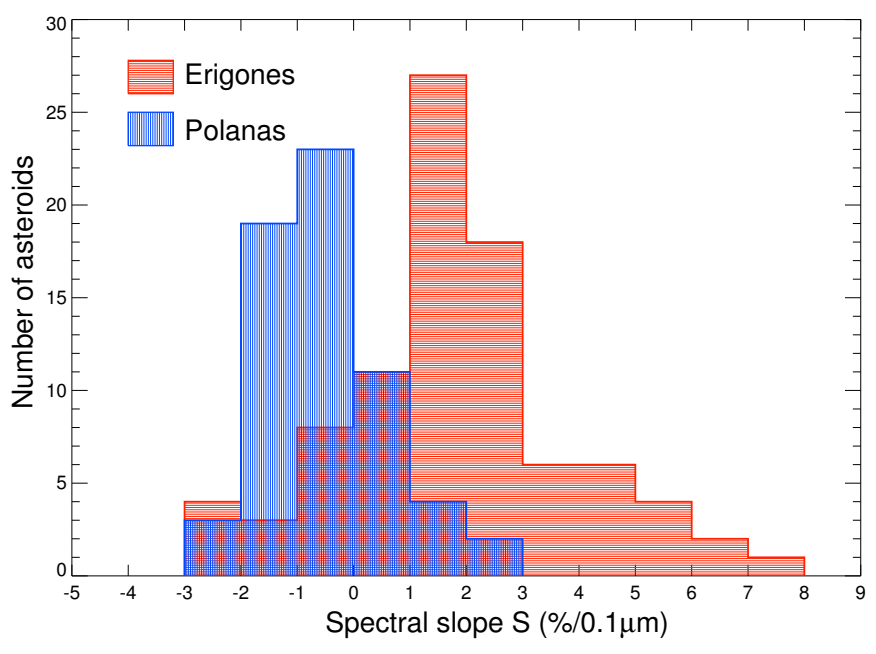

Fig. 4. Histogram showing the distribution for the values of the spectral slope for the primitive asteroids in the Erigone family (red). The distribution of the slopes of the asteroids of the Polana family from de León et al. (2016) is shown in blue as a comparison.

two distributions are significantly different, with the asteroids of the Erigone family showing redder spectral slopes in general.

\subsection{Aqueous alteration}

Several studies (Vilas 1994; Fornasier et al. 1999; Carvano et al. 2003; Rivkin 2012; Fornasier et al. 2014) showed that a considerable number of main belt primitive asteroids present an absorption feature around $0.7 \mu \mathrm{m}$ attributed to charge transfer transitions in oxidized iron (Vilas \& Gaffey 1989; Vilas 1994; Barucci et al. 1998), which is indicative of, or associated with, 


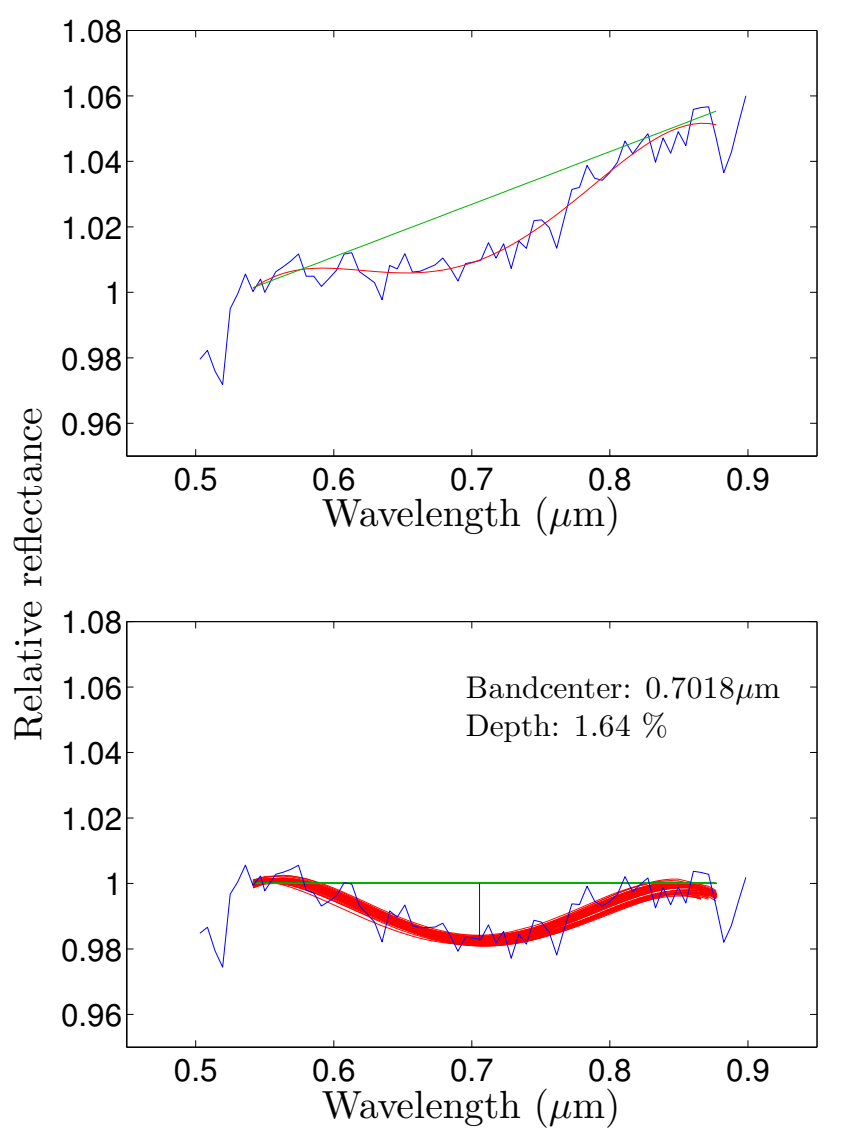

Fig. 5. Example figure of the process followed to compute the central wavelength position and depth of the $0.7 \mu \mathrm{m}$ absorption band on the spectra of asteroid (72384). The top panel shows the straight line (green) used to remove the continuum from the fitted absorption band (red curve). The bottom panel shows the result of this continuum removal and the iterative procedure using a Monte Carlo model to compute the band parameters (see text for details).

aqueous alteration in the surface of these objects (i.e., presence of hydrated minerals). Fornasier et al. (2014) showed that (163) Erigone, the parent body of the family, presents this particular absorption feature at $0.7 \mu \mathrm{m}$, with a depth of $2.2 \pm 0.1 \%$ with respect to the continuum. To search for said absorption feature among our Erigone family member spectra, we followed the procedure described in Carvano et al. (2003) with some minor adjustments. For objects that showed this feature, we characterize the central wavelength position and depth.

The first step was to compute the continuum of the absorption band by fitting a straight line tangent to the spectra at two positions, $0.54-0.56 \mu \mathrm{m}$ and $0.86-0.88 \mu \mathrm{m}$, which are the limits of the $0.7 \mu \mathrm{m}$ absorption band (green line in the top panel of Fig. 5). We tested slightly different ranges to compute the continuum, finding no significant changes in our results. Then, we fitted the spectrum in this interval using a fourth-order spline (red curve in the top panel of Fig. 5).

The final step was to remove the continuum by dividing the spline fit by the straight line we previously obtained (bottom panel of Fig. 5). To compute the depth and central wavelength position of the absorption band and their corresponding errors, we ran a Monte Carlo model with 1000 iterations, randomly removing ten points from the spectrum in the range from 0.54 to $0.88 \mu \mathrm{m}$ at each iteration, then repeating the procedure. The band depth is computed as the difference, in $\%$, between a reflectance

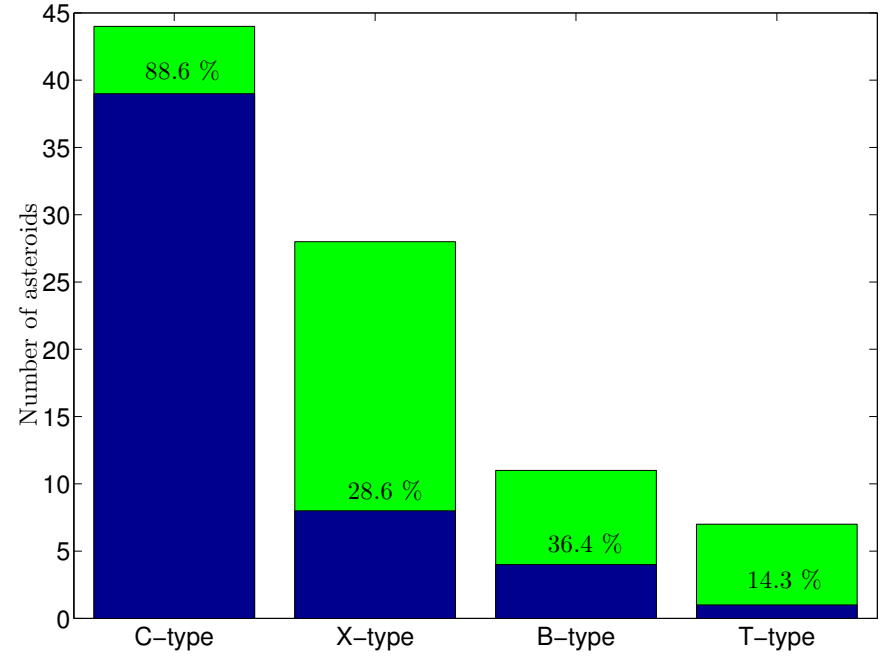

Fig. 6. Percentage of asteroids showing the $0.7 \mu \mathrm{m}$ absorption band (dark blue) for each primitive taxonomic class (green).

value of 1 and the reflectance value corresponding to the central wavelength position. The final values for the band depth and central wavelength are computed as the mean values obtained for the full Monte Carlo run, and the errors are the corresponding $1 \sigma$ standard deviations. The criterion for deciding whether an object showed an aqueous alteration band was to rule out objects that presented bands with a depth smaller than $1 \%$ or relative errors in the computation of the depth larger than $15 \%$. The detection threshold of $1 \%$ corresponds to the peak-to-peak scatter in our spectra, which seems to be a better indicator of the spectrum quality than the calculated signal-to-noise ratio.

We have to note that there are some cases in which the detected bands have depths that are higher than expected. In these cases (in particular, that of asteroid 210564), high depth values arise because the absorption band is quite broad and the limiting regions are narrow and not perfectly defined. In the specific case of asteroid 210564 , the band depth might vary from $\sim 9 \%$ to $\sim 5 \%$ if we were to change the continuum-fitting upper limits. This is because when we move the upper limit below $0.88 \mathrm{mi}$ crons, we might fall already inside the band, which would produce heavy band depth shifts with small error bars. These special cases might reveal the limitations of our data (if we had information over $0.9 \mu \mathrm{m}$, the band limits would be clearer).

In Table C. 3 we indicate for each asteroid the taxonomical classification, whether it has an absorption band at $0.7 \mu \mathrm{m}$ (YES/NO), and the center and depth of the band, when present. We found that 52 of the 90 primitive asteroids present an absorption band at $0.7 \mu \mathrm{m}$ and that this band is present regardless of the specific primitive spectral class. Figure 6 shows the proportion of asteroids with a hydration band for each primitive class. Almost all C-type asteroids present the hydration feature $(\sim 88 \%)$. This proportion is progressively smaller for B-types $(\sim 36 \%)$, X-types $(\sim 28.5 \%)$, and T-types $(\sim 14 \%)$. This decreasing trend in our results agrees with previous studies of the relative incidence of this feature in asteroids distributed throughout the main belt. Vilas (1994) found an incidence of $47.7 \%$ in C-type asteroids and $33 \%$ in B-types, while Fornasier et al. (2014) found an incidence of $50.7 \%$ in C-types and a $9.8 \%$ in B-types. The proportion of C-type asteroids showing the $0.7 \mu \mathrm{m}$ absorption band is significantly larger in the Erigone family, with about $87 \%$ of the asteroids. 
That there is a primitive collisional family, with all the observed objects located between 2.3 and 2.4 AU and with the majority of its members showing the $0.7 \mu \mathrm{m}$ hydration band, agrees well with the results presented by Fornasier et al. (2014), who suggested that the aqueous alteration processes dominate in primitive asteroids located between 2.3 and 3.1 AU. Moreover, they stated that the proportion of hydrated primitive objects in the region where the Erigone family is located is 64\%, which agrees very well with the proportion of hydrated objects we have found in our sample, which is $57.7 \%$ (52 out of 90).

We found no significant correlations between the band depths or the band centers and the taxonomical class, the asteroid orbital parameters, the albedo, or the size of the objects. A similar lack of correlations was found by Carvano et al. (2003) and Fornasier et al. (2014) in their respective studies for asteroids throughout the whole main belt. Additionally, the mean values we found for the band depth, $2.9 \pm 1.5 \%$, and the band center position, $7053 \pm 160 \AA$, agree excellently well with those obtained by Fornasier et al. (2014): $2.8 \pm 1.2 \%$ for the band depth and $6914 \pm 148 \AA$ for the band center. These results suggest that the values obtained for the parameters used to characterize the $0.7 \mu \mathrm{m}$ hydration band are independent of the location of the asteroids in the main belt.

\subsection{Comparison with spectra of (101955) Bennu and (162173) Ryugu}

As we described in Sect. 1, the aim of characterizing the Erigone primitive family is that together with the Polana, Clarissa, and Sulamitis families and the low-albedo and low-inclination background asteroids, they are the most likely sources of the two NEAs that are the targets of the OSIRIS-REx and Hayabusa 2 sample-return missions: (101955) Bennu and (162173) Ryugu. Spectroscopic and photometric observations of these asteroids suggest that they are composed of primitive materials, pointing to an origin in the aforementioned populations, which is reinforced by the results of dynamical simulations (Campins et al. 2010, 2013; Bottke et al. 2015).

Spectral comparison might shed light upon the origins of the two asteroids, and we therefore compared the available visible spectra of asteroids (101955) Bennu and (162173) Ryugu with the data obtained in this work.

For (162173) Ryugu, several references in the literature show visible spectra of this small $(\sim 800 \mathrm{~m})$, low-albedo $\left(p_{V}=0.07\right)$ NEA. A first spectrum from Binzel et al. (2001) shows an ultraviolet drop-off in reflectance shortward of $0.65 \mu \mathrm{m}$ and provides a classification of Cg-type. Two other visible spectra were presented in Vilas (2008), obtained in July and September 2007. These two spectra were different from each other and also different from the spectrum reported by Binzel et al. (2001), which showed no ultraviolet drop-off. The spectrum obtained in July showed an absorption band at $0.7 \mu \mathrm{m}$ and a red spectral slope, while the one obtained in September, with a much higher signalto-noise ratio, presented a neutral slope and showed a marginal, very shallow absorption centered near $0.6 \mu \mathrm{m}$. According to Vilas (2008), these differences suggest that the surface of the asteroid covers the conjunction of two different geological units. A comparison between these three spectra can be seen in the left panel of Fig. 3 from Campins et al. (2013). Additional rotationally resolved visible spectra of (162173) Ryugu were presented by Lazzaro et al. (2013), Moskovitz et al. (2013), and Sugita et al. (2013), all of them compatible with a C-type classification and showing no absorption feature at $0.7 \mu \mathrm{m}$ and a spectral
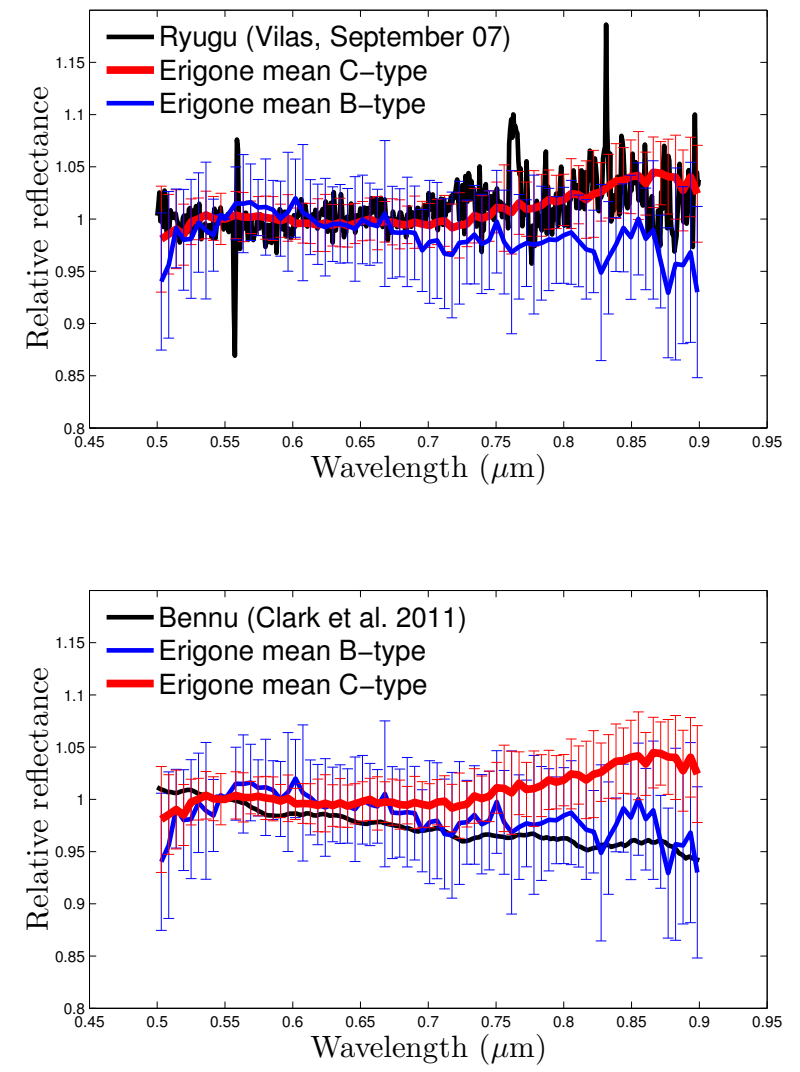

Fig. 7. Comparison between the September 2007 (Vilas 2008) visible spectrum of Ryugu in the top panel and the visible spectrum of (101955) Bennu from Clark et al. (2011) in the bottom panel, with the mean spectra of the C-type (red) and B-type (blue) asteroids in the Erigone family. The standard deviation of the mean $( \pm 1 \sigma)$ is shown with vertical lines.

slope similar to the September 2007 spectrum from Vilas (2008), making the suggestion of two different surfaces very unlikely. Because it is the spectrum with the highest signal-to-noise ratio, we therefore selected it to perform our spectral comparison. The upper panel of Fig. 7 shows the September 2007 (Vilas 2008) spectrum of Ryugu compared to the mean visible spectra of the asteroids of the Erigone family classified as C-types (and subclasses) in red, and those classified as B-types in blue. The visible spectrum of Ryugu agrees well with the mean spectrum of C-type asteroids in the Erigone family, even though it does not show the $0.7 \mu \mathrm{m}$ absorption feature.

Even if the signal-to-noise ratio is quite poor, we computed both the wavelength central position and band depth of the $0.7 \mu \mathrm{m}$ absorption band present in the July 2007 spectrum of Ryugu (Vilas 2008). Our calculations yielded a depth of $11.7 \pm 1.3 \%$ and a band center of $6870 \pm 55 \AA$. The absorption band depth and band center are very different from the mean values computed by Fornasier et al. (2014) and also from those computed in this work for the asteroids in the Erigone family (see Sect. 3.2). Together with the absence of the $0.7 \mu \mathrm{m}$ absorption band in all the subsequent visible spectra of Ryugu obtained by other authors, this suggests that the absorption band observed in the July 2007 spectrum from Vilas (2008) might be due to some artifact.

The only available visible data for the primitive $\left(p_{V}=0.043\right)$ and rather small ( 500 m) NEA (101955) Bennu are found in Clark et al. (2011) and Hergenrother et al. (2013). Based on these studies, Bennu is classified as a B-type asteroid in the Bus \& Binzel (2002) taxonomy. In addition, Binzel et al. (2015) 
reported spectra obtained in the NIR range that showed spectral variability, which indicates a C-type class according to DeMeo et al. (2009). As has been shown in Clark et al. (2010) and de León et al. (2012), asteroids classified as B-types in the visible can present considerable slope variation in the NIR, from negative blue slopes to positive redder ones. The lower panel of Fig. 7 shows the visible spectrum of Bennu (black) compared to the mean visible spectra of the asteroids of the Erigone family classified as C-types (and subclasses) in red and those classified as B-types in blue, as observed in this work. From a visual inspection, the visible spectrum of Bennu seems to marginally show the presence of the hydration feature $0.7 \mu \mathrm{m}$. We followed the same approach as in the previous section to study the presence of a possible aqueous alteration band. Our calculations yielded the presence of a band centered at $7486 \pm 76 \AA$, with a depth of $0.96 \pm 0.02 \%$. This depth is slightly below the threshold $(1 \%)$ established for a positive detection, and the wavelength position of the band center is significantly different from the mean value found for the family $(7053 \pm 160 \AA)$. Therefore, we rule out the presence of this hydration band in the spectrum of Bennu.

\section{Discussion}

No previous spectroscopic studies have been performed until now on the Erigone primitive collisional family, with only two asteroids that were previously classified using visible spectroscopy: (163) Erigone, and (571) Dulcinea. We clearly showed that the asteroids we studied here are spectroscopically consistent with the hypothesis of a common parent body, and that this parent body is (163) Erigone, classified as a C-type asteroid. Moreover, (163) Erigone shares the particular spectroscopic feature at $0.7 \mu \mathrm{m}$ with most of the family members. Another primitive collisional family in the inner belt, the Polana family, which together with Erigone, Sulamitis, and Clarissa, represents the four primitive families in the inner belt, has recently been studied by several authors (Walsh et al. 2013; Milani et al. 2014; Dykhuis \& Greenberg 2015; de León et al. 2016; Pinilla-Alonso et al. 2016). Comparing our results on the Erigone family with those obtained from de León et al. (2016) for the Polana family, we observe two main differences.

a) The Erigone family presents a different distribution of taxonomical classes from that of the Polana family, referred to as the Polana-Eulalia complex in de León et al. (2016). In our sample, we found $44 \mathrm{C}$-type objects, $28 \mathrm{X}$-types, $11 \mathrm{~B}$-types, and 7 T-types, plus 13 interlopers (S-types and L-types). The mean spectra for each class are clearly differentiated, especially in the $\mathrm{X}$ and $\mathrm{T}$ classes (see Fig. 8). In contrast, we found mainly C- (51\%) and B-types (42\%) in the Polana family, with a few X-types (5\%) and only one S-type, with the mean spectra for the C-, B-, and X-types presenting similar values (de León et al. 2016). In addition, the slope distribution for the objects in the Erigone family is clearly redder than that of the Polana family, as can be seen in Fig. 4, which is mainly due to the larger fraction of X-types found in the former.

b) The majority of the primitive asteroids in the Erigone family (52 out of 90) show evidence of aqueous altered minerals on their surfaces. We conducted the same analysis as that described in Sect. 3.2 for the data in de León et al. (2016) and found that according to our criterion, only one object in the Polana family (asteroid 29626) showed the $0.7 \mu \mathrm{m}$ absorption feature, with a band depth of $1.15 \pm 0.11 \%$ and a band center of $7300 \pm 211 \AA$. This difference in the

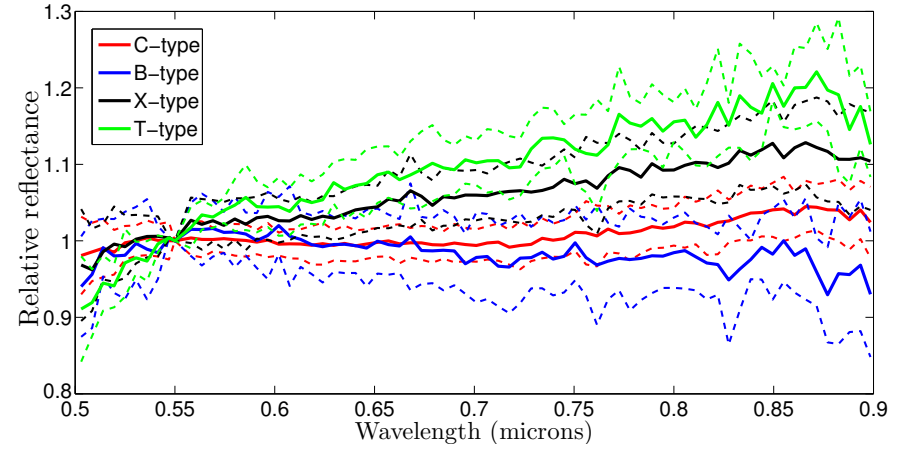

Fig. 8. Computed mean spectra of the asteroids in the Erigone family classified as C-types (red), B-types (blue), X-types (black), and T-types (green). The mean spectrum for each class is plotted as a thick line, while the $\pm 1 \sigma$ of the mean is shown with dashed lines. The different mean spectra are well differentiated from one another.

two families is well explained by their two parent bodies. Asteroid (142) Polana shows no signs at all of the hydration feature at $0.7 \mu \mathrm{m}$. On the other hand, (163) Erigone, the parent body of the Erigone family, shows evidence of aqueous alteration. Therefore, the presence of the hydration feature in the spectra of most of its family members is somewhat expected. An interesting explanation for the difference in hydration between the two families was presented by Matsuoka et al. (2015). They proposed that space weathering effects on C-type asteroids tend to make the $0.7 \mu \mathrm{m}$ absorption feature less deep. Space weathering processes might have removed the aqueous alteration band in the Polana family, since the Polana family is older than Erigone: according to Bottke et al. (2015), Erigone is $130 \pm 30$ Myr old, while Polana (referred to as New Polana in their paper) and Eulalia are $1400 \pm 150$ Myr old, and 830 +3700 Myr old, respectively.

To determine the likelihood that the Erigone family is the source for asteroids (101955) Bennu and (162173) Ryugu, we compared visible spectra of the two objects with the mean spectra of the primitive asteroids in the family. In our sample, we found approximately $56 \%$ of non-interlopers showing evidence of aqueous alteration. According to Bottke et al. (2015), there is little to no chance for the smaller families (Erigone, Clarissa, and Sulamitis) to be the source of these two asteroids:

- (101955) Bennu: Bennu is classified as a B-type asteroid in the visible wavelength range, according to Clark et al. (2011) and Hergenrother et al. (2013). Even if the fraction of B-type asteroids in the Erigone family is small, as is the probability of Bennu coming from the Erigone family (Bottke et al. 2015), the spectral comparison between the spectrum of Bennu and the mean spectrum of the B-type asteroids is compatible with a possible origin in the Erigone family.

- (162173) Ryugu: Vilas (2008) reported an aqueous alteration band around $0.7 \mu \mathrm{m}$ in one of the three visible spectra obtained for Ryugu (July 2007, see their Fig. 4). Following the same approach we used for our aqueous alteration study, the depth of the band we computed in the July 2007 spectrum from Vilas (2008), $11.7 \pm 1.3 \%$, is far from the medium depth of the aqueous altered asteroids in Fornasier et al. (2014) $(2.8 \pm 1.2 \%)$ and also in this work $(2.9 \pm 1.5 \%)$. However, from the spectral comparison, the possibility of Ryugu originating in the Erigone family should not be discarded. Ryugu is a C-type asteroid, and the most abundant spectral classes in the Erigone family are C-type asteroids and subclasses $(\mathrm{Cb}$, 
$\mathrm{Cg}, \mathrm{Ch}$, and $\mathrm{Cgh}$ ). This would point to a possible origin of Ryugu in the Erigone family, even if all the visible spectra of this asteroid obtained by different authors show no $0.7 \mu \mathrm{m}$ absorption band.

Since the Polana family, suggested in Bottke et al. (2015) as the most probable source for both NEAs, does not show signs of aqueous altered asteroids (Walsh et al. 2013; Dykhuis \& Greenberg 2015; de León et al. 2016), the Erigone family cannot be discarded as a possible source for the two NEAs. More observations of (101955) Bennu and (162173) Ryugu need to be made to check whether the $3 \mu \mathrm{m}$ absorption feature is present or not, definitely indicating evidence of aqueous alteration, and confirming or ruling out the chances for Erigone being the source of these objects.

\section{Conclusions}

We studied a total of 103 visible spectra of asteroids in the Erigone primitive family. We observed 101 of these asteroids with the $10.4 \mathrm{~m}$ Gran Telescopio Canarias. These objects have never been observed before with spectroscopy. We added the visible spectra of (163) Erigone and (571) Dulcinea from the SMASS database for completeness.

We showed that the slope distribution and the taxonomic classification of the asteroids agree with the assumption of a primitive family. Most of the observed objects are C-types, highly consistent with the classification of (163) Erigone, supporting its status as the parent body of the family. In terms of taxonomical distribution, $42 \%$ of the asteroids in our survey are classified as C-type objects, $28 \%$ as X-types, $11 \%$ as B-types, $7 \%$ as T-types, and $13 \%$ of the objects as non-primitive types, most likely interlopers (S-types and subclasses, and L-types).

In addition, the study of aqueous alteration performed on the group of primitive objects shows a high number of hydrated asteroids. The C-type class shows the largest number of hydrated asteroids, namely $\sim 86 \%$. The other primitive classes, also showing the hydration band, present a much smaller fraction: B-type objects, $\sim 36 \%$, X-type objects, $\sim 28 \%$, and T-type, $\sim 14 \%$. This distribution of the hydration as a function of the taxonomical class agrees with the one in Fornasier et al. (2014) for asteroids in the main belt.

Based on the spectral comparison alone and as for the Polana family that was studied in de León et al. (2016), we cannot discard the possibility of Erigone being the source family for the NEAs (101955) Bennu and (162173) Ryugu. The spectral classes present in the family are compatible with the taxonomic classification of the two asteroids. Future research should include further spectroscopic study, both in the visible and nearinfrared regions, of the other primitive families in the inner belt, such as Sulamitis and Clarissa, to completely rule them out as the possible sources of asteroids (101955) Bennu and (162173) Ryugu.

Acknowledgements. D.M. gratefully acknowledges the Spanish Ministry of Economy and Competitiveness (MINECO) for the financial support received in the form of a Severo-Ochoa Ph.D. fellowship, within the Severo-Ochoa International Ph.D. Program. D.M., J.d.L., J.L., and V.L. acknowledge support from the project AYA2012-39115-C03-03 and ESP2013-47816-C42-P (MINECO). J.d.L. acknowledges support from the Insituto de Astrofísica de Canarias. H.C. acknowledges support from NASA's Near-Earth Object Observations program and from the Center for Lunar and Asteroid Surface Science funded by NASA's SSERVI program at the University of Central Florida. The authors gratefully acknowledge the referee, Sonia Fornasier, for her comments and suggestions. The results obtained in this paper are based on observations made with the Gran Telescopio Canarias (GTC), installed in the Spanish Observatorio del Roque de los Muchachos of the Instituto de Astrofísica de Canarias, in the island of La Palma.

\section{References}

Barucci, M. A., Doressoundiram, A., Fulchignoni, M., et al. 1998, Icarus, 132, 388

Bevington, P. R., \& Robinson, D. K. 1992, Data reduction and error analysis for the physical sciences (New-York, USA: McGraw-Hill Companies)

Binzel, R. P., \& Xu, S. 1993, Science, 260, 186

Binzel, R. P., Harris, A. W., Bus, S. J., \& Burbine, T. H. 2001, Icarus, 151, 139

Binzel, R. P., DeMeo, F. E., Burt, B. J., et al. 2015, Icarus, 256, 22

Bottke, W. F., Morbidelli, A., Jedicke, R., et al. 2002, Icarus, 156, 399

Bottke, W. F., Vokrouhlický, D., Walsh, K. J., et al. 2015, Icarus, 247, 191

Bus, S. J., \& Binzel, R. P. 2002, Icarus, 158, 146

Campins, H., Morbidelli, A., Tsiganis, K., et al. 2010, ApJ, 721, L53

Campins, H., de León, J., Morbidelli, A., et al. 2013, AJ, 146, 26

Carvano, J. M., Mothé-Diniz, T., \& Lazzaro, D. 2003, Icarus, 161, 356

Cepa, J. 2010, in Highlights of Spanish Astrophysics V, eds. J. M. Diego, L. J. Goicoechea, J. I. González-Serrano, \& J. Gorgas (Springer), 15

Cepa, J., Aguiar, M., Escalera, V. G., et al. 2000, in Optical and IR Telescope Instrumentation and Detectors, eds. M. Iye, \& A. F. Moorwood, SPIE Conf. Ser., 4008, 623

Clark, B. E., Ziffer, J., Nesvorny, D., et al. 2010, J. Geophys. Res. (Planets), 115, 6005

Clark, B. E., Binzel, R. P., Howell, E. S., et al. 2011, Icarus, 216, 462

de León, J., Pinilla-Alonso, N., Campins, H., Licandro, J., \& Marzo, G. A. 2012, Icarus, 218, 196

de León, J., Pinilla-Alonso, N., Delbo, M., et al. 2016, Icarus, 266, 57

DeMeo, F. E., \& Carry, B. 2013, Icarus, 226, 723

DeMeo, F. E., Binzel, R. P., Slivan, S. M., \& Bus, S. J. 2009, Icarus, 202, 160

Dykhuis, M. J., \& Greenberg, R. 2015, Icarus, 252, 199

Fornasier, S., Lantz, C., Barucci, M. A., \& Lazzarin, M. 2014, Icarus, 233, 163

Fornasier, S., Lazzarin, M., Barbieri, C., \& Barucci, M. A. 1999, A\&AS, 135, 65

Hergenrother, C. W., Nolan, M. C., Binzel, R. P., et al. 2013, Icarus, 226, 663

Hirayama, K. 1918, AJ, 31, 185

Howell, E. S., Rivkin, A. S., Vilas, F., et al. 2011, in EPSC-DPS Joint Meeting 2011, 637

Landolt, A. U. 1992, AJ, 104, 340

Lauretta, D. S., Drake, M. J., Benzel, R. P., et al. 2010, Meteorit. Planet. Sci. Suppl., 73, 5153

Lazzaro, D., Barucci, M. A., Perna, D., et al. 2013, A\&A, 549, L2

Luu, J. X., \& Jewitt, D. C. 1990, AJ, 99, 1985

Masiero, J. R., Mainzer, A. K., Grav, T., et al. 2011, ApJ, 741, 68

Matsuoka, M., Nakamura, T., Kimura, Y., et al. 2015, Icarus, 254, 135

Milani, A., Cellino, A., Knežević, Z., et al. 2014, Icarus, 239, 46

Moskovitz, N. A., Abe, S., Pan, K.-S., et al. 2013, Icarus, 224, 24

Nesvorny, D. 2012, NASA Planetary Data System, 189

Pinilla-Alonso, N., de León, J., Walsh, K. J., et al. 2016, Icarus, submitted

Popescu, M., Birlan, M., \& Nedelcu, D. A. 2012, A\&A, 544, A130

Rivkin, A. S. 2012, Icarus, 221, 744

Sugita, S., Kuroda, D., Kameda, S., et al. 2013, in Lunar and Planetary Inst. Technical Report, 44, 2591

Tsuda, Y., Yoshikawa, M., Abe, M., Minamino, H., \& Nakazawa, S. 2013, Acta Astron., 91, 356

Vilas, F. 1994, Icarus, 111, 456

Vilas, F. 2008, AJ, 135, 1101

Vilas, F., \& Gaffey, M. J. 1989, Science, 246, 790

Vokrouhlický, D., Brož, M., Bottke, W. F., Nesvorný, D., \& Morbidelli, A. 2006, Icarus, 182,118

Walsh, K. J., Delbó, M., Bottke, W. F., Vokrouhlický, D., \& Lauretta, D. S. 2013, Icarus, 225, 283

Zappala, V., \& Cellino, A. 1994, in Asteroids, Comets, Meteors 1993, eds. A. Milani, M. di Martino, \& A. Cellino, IAU Symp., 160, 395

Zappala, V., Cellino, A., Farinella, P., \& Knezevic, Z. 1990, AJ, 100, 2030 


\section{Appendix A: Primitive spectra}

We present here the visible spectra of the primitive asteroids (a total of 90). The spectra are normalized to unity at $0.55 \mu \mathrm{m}$ and binned (see Sect. 2.2). Visible spectrum of asteroid (163) Erigone has been taken from the SMASS-II survey.

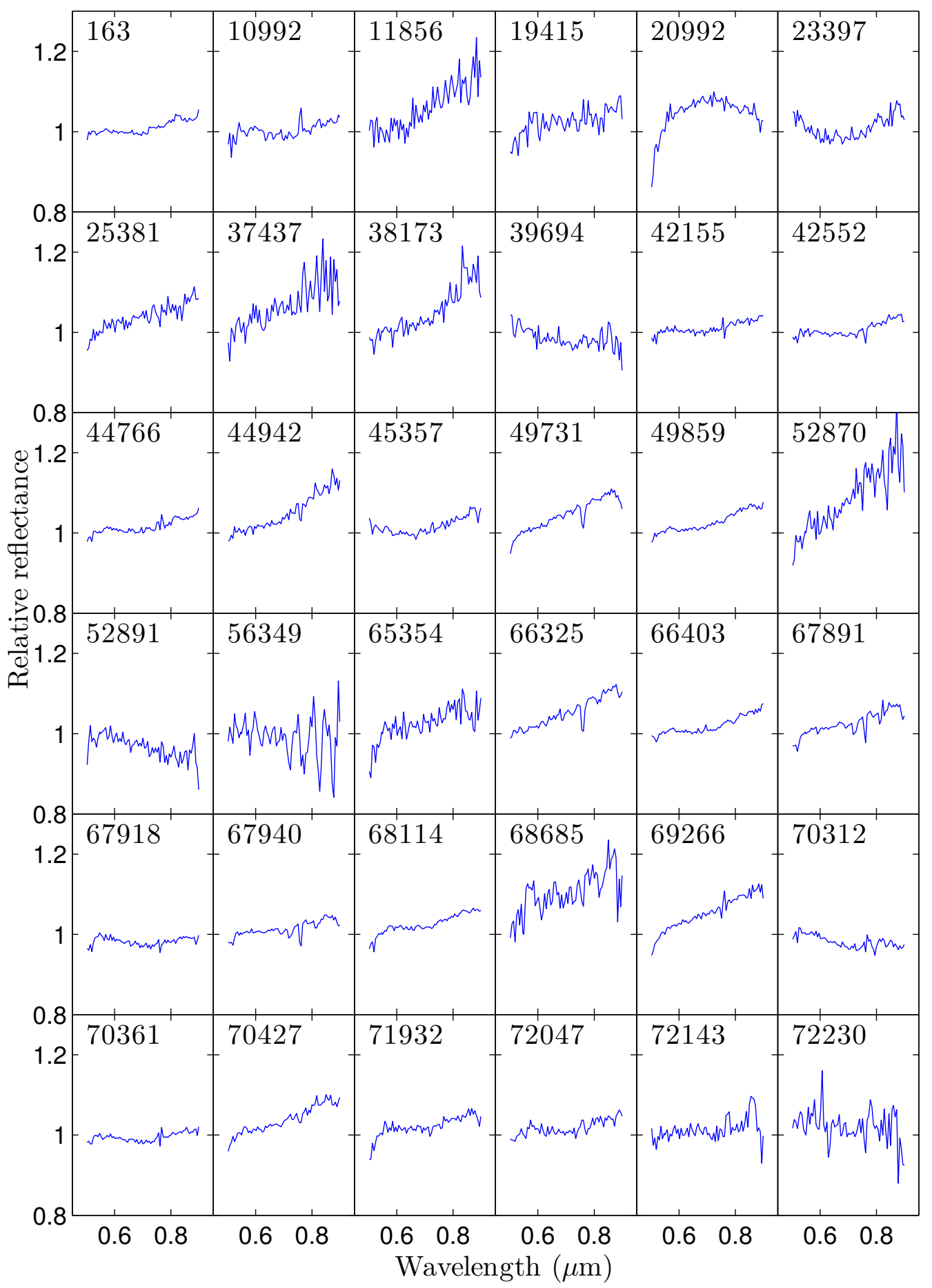

Fig. A.1. Visible spectra of the primitive asteroids. Spectra are normalized to unity at $0.55 \mu \mathrm{m}$. 
D. Morate et al.: Visible spectroscopy of Erigone collisional family

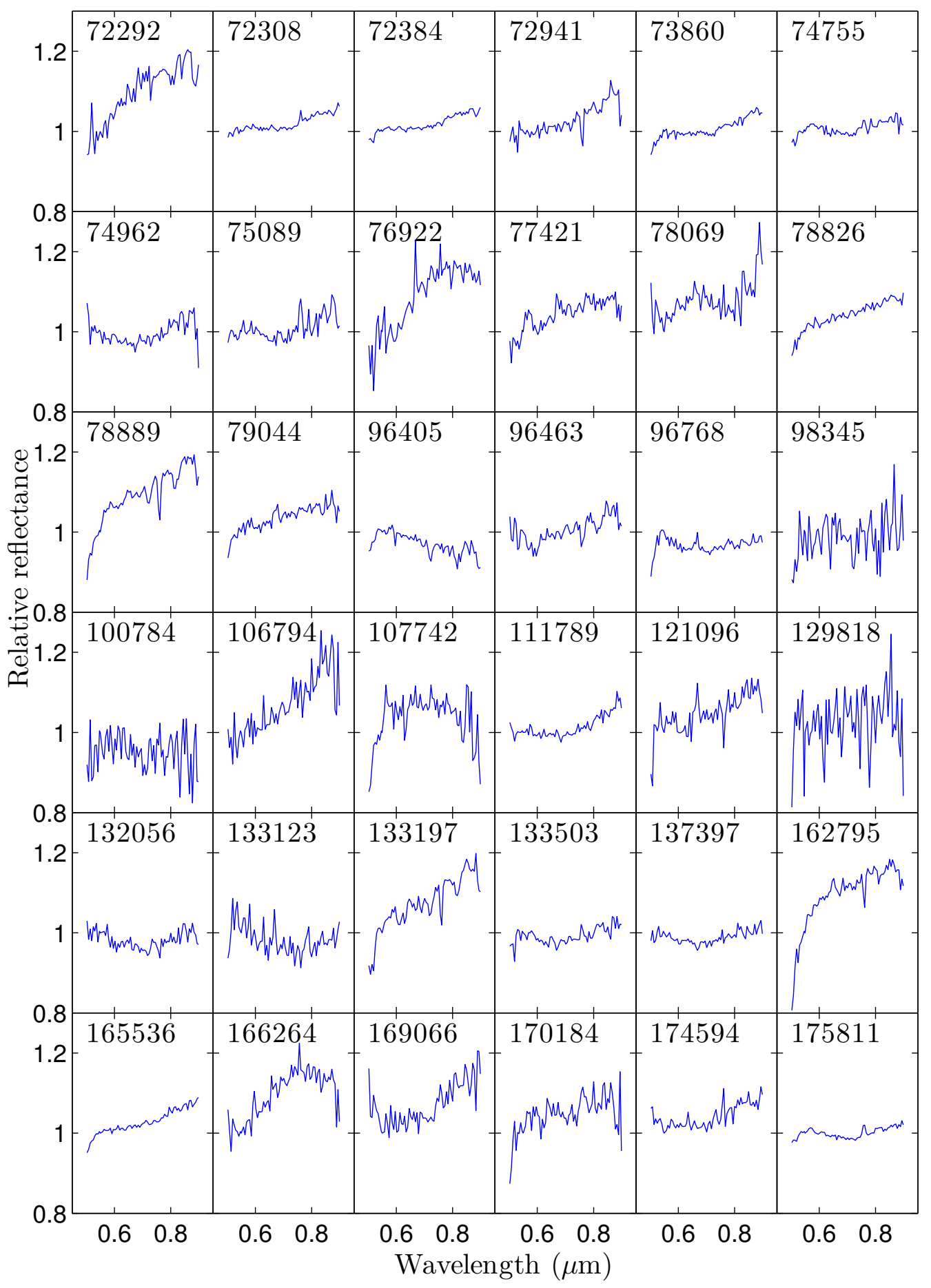

Fig. A.1. continued. 


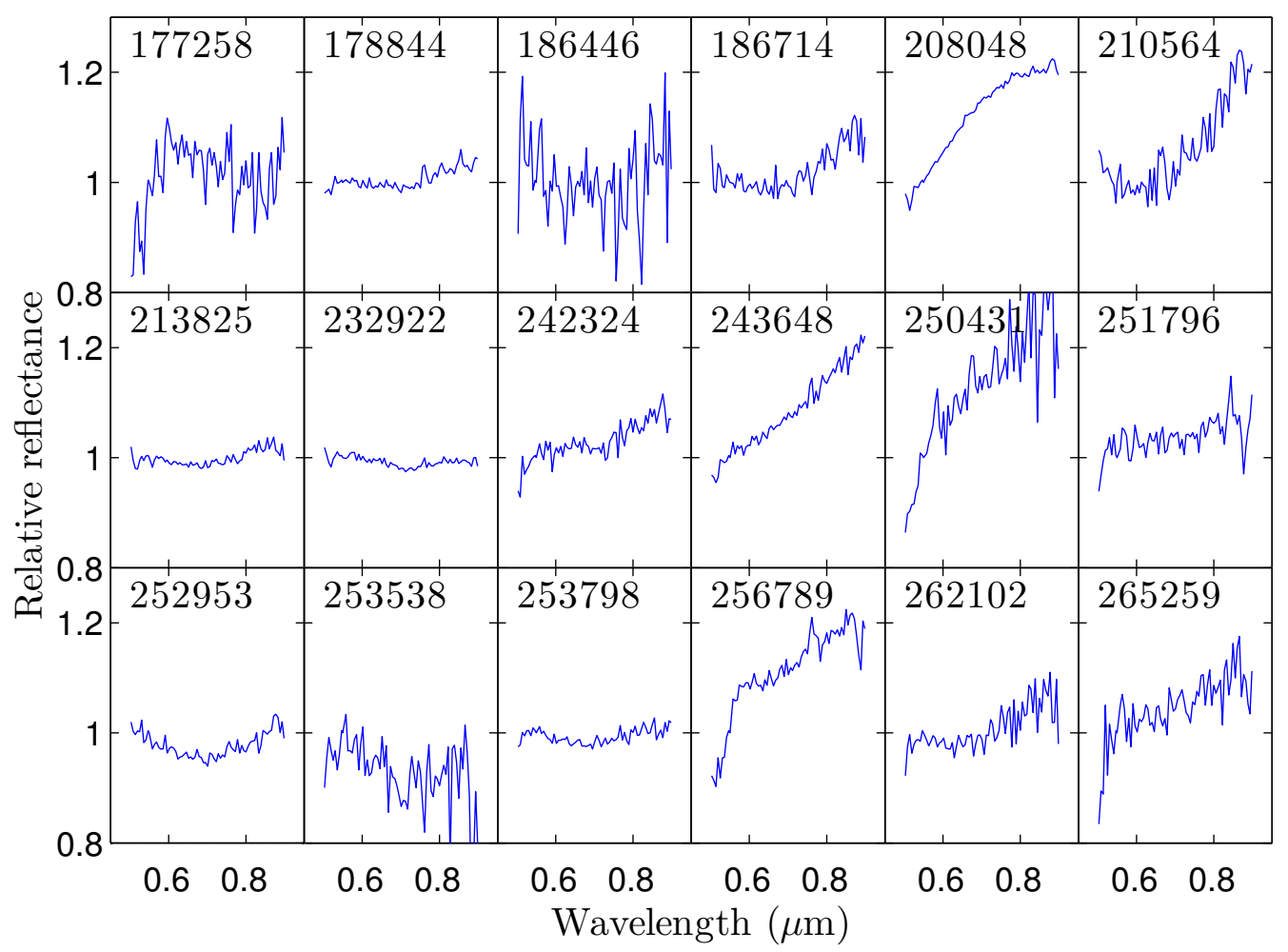

Fig. A.1. continued.

\section{Appendix B: Non-primitive spectra}

We present here the visible spectra of the non-primitive asteroids (a total of 13). The spectra are normalized to unity at $0.55 \mu \mathrm{m}$ and binned (see Sect. 2.2). Visible spectrum of asteroid (571) Dulcinea has been taken from the SMASS-II survey.

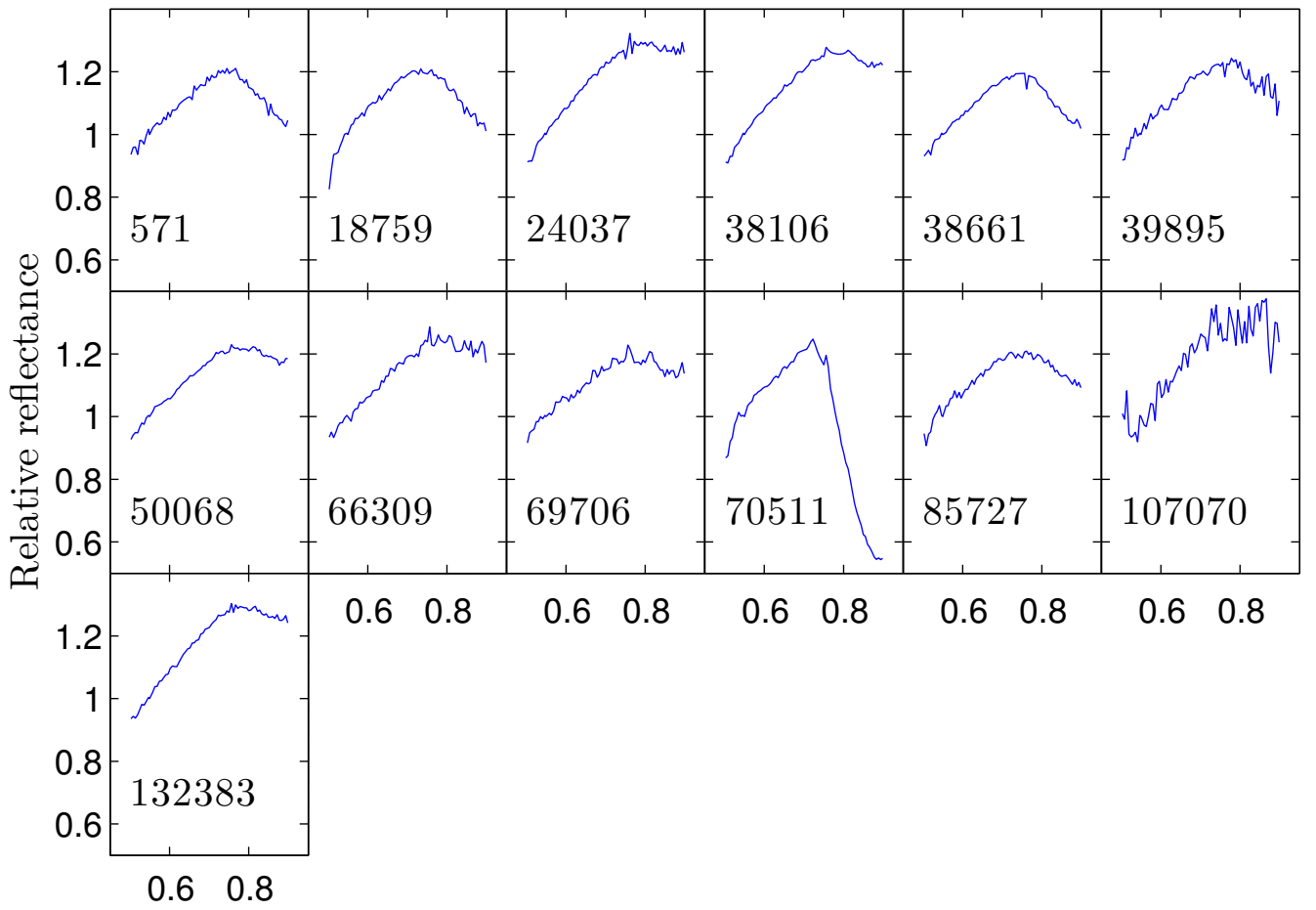

Wavelength $(\mu \mathrm{m})$

Fig. B.1. Visible spectra of the non-primitive asteroids. Spectra are normalized to unity at $0.55 \mu \mathrm{m}$. 
D. Morate et al.: Visible spectroscopy of Erigone collisional family

\section{Appendix C: Additional tables}

Table C.1. Observational circumstances of the asteroids presented in this paper.

\begin{tabular}{|c|c|c|c|c|c|c|}
\hline Object & Date & UT start & Airmass & Exposure time (s) & SAs & Seeing $\left({ }^{\prime \prime}\right)$ \\
\hline 10992 & 2014-08-16 & $05: 46$ & 1.117 & $3 \times 200$ & 5 & 0.9 \\
\hline 11856 & $2015-01-12$ & 01:10 & 1.482 & $3 \times 250$ & 2 & 2.0 \\
\hline 18759 & $2014-12-17$ & $22: 35$ & 1.149 & $3 \times 250$ & 1 & 2.0 \\
\hline 19415 & 2014-09-18 & $22: 23$ & 1.310 & $3 \times 300$ & 4,6 & 1.0 \\
\hline 20992 & 2014-12-17 & $03: 13$ & 1.248 & $3 \times 250$ & 2 & 1.9 \\
\hline 23397 & $2015-01-14$ & $03: 51$ & 1.292 & $3 \times 300$ & 1,3 & 1.5 \\
\hline 24037 & 2014-12-18 & 01:27 & 1.305 & $2 \times 200$ & 1 & 2.7 \\
\hline 25381 & $2014-12-18$ & $00: 22$ & 1.352 & $3 \times 500$ & 1 & 2.0 \\
\hline 37437 & $2014-12-17$ & $22: 04$ & 1.335 & $3 \times 500$ & 1 & 2.5 \\
\hline 38106 & 2014-10-14 & $03: 43$ & 1.033 & $3 \times 400$ & 1,2 & 1.0 \\
\hline 38173 & 2014-12-15 & $23: 46$ & 1.554 & $3 \times 300$ & 1,2 & 2.0 \\
\hline 38661 & 2014-09-13 & 03:04 & 1.257 & $3 \times 200$ & 1,6 & 0.9 \\
\hline 39694 & 2014-09-19 & $00: 18$ & 1.198 & $3 \times 400$ & 4,6 & 0.9 \\
\hline 39895 & 2014-09-15 & 03:38 & 1.507 & $3 \times 300$ & 1,6 & 0.8 \\
\hline 42155 & 2014-09-13 & $03: 36$ & 1.095 & $3 \times 200$ & 1,6 & 0.8 \\
\hline 42552 & 2014-09-15 & $02: 50$ & 1.513 & $3 \times 250$ & 1,6 & 0.9 \\
\hline 44766 & $2014-12-17$ & $03: 58$ & 1.039 & $3 \times 200$ & 2 & 1.3 \\
\hline 44942 & $2015-01-14$ & 03:29 & 1.496 & $3 \times 200$ & 1,3 & 1.5 \\
\hline 45357 & $2015-01-14$ & $04: 22$ & 1.416 & $3 \times 300$ & 1,3 & 1.5 \\
\hline 49731 & 2015-01-14 & $04: 46$ & 1.920 & $3 \times 200$ & 1,3 & 1.9 \\
\hline 49859 & 2015-05-04 & $23: 37$ & 1.300 & $3 \times 180$ & 3,7 & 1.5 \\
\hline 50068 & 2015-05-05 & $00: 10$ & 1.131 & $3 \times 180$ & 3,7 & 1.5 \\
\hline 52870 & 2015-01-19 & $05: 57$ & 1.124 & $3 \times 500$ & 3 & 2.0 \\
\hline 52891 & $2015-01-15$ & 03:53 & 1.197 & $3 \times 500$ & 3 & 1.2 \\
\hline 56349 & $2015-01-15$ & $04: 29$ & 1.163 & $3 \times 500$ & 3 & 1.1 \\
\hline 65354 & $2014-12-13$ & 01:05 & 1.048 & $3 \times 500$ & 2 & 1.5 \\
\hline 66309 & 2014-10-14 & $02: 34$ & 1.456 & $3 \times 250$ & 1,2 & 1.4 \\
\hline 66325 & $2014-10-13$ & 04:20 & 1.847 & $3 \times 300$ & 1,2 & 1.5 \\
\hline 66403 & 2014-09-14 & 01:35 & 1.110 & $3 \times 200$ & 1,6 & 0.9 \\
\hline 67891 & 2014-09-15 & 02:09 & 1.684 & $3 \times 200$ & 1,6 & 1.0 \\
\hline 67918 & 2014-09-13 & 02:39 & 1.214 & $3 \times 200$ & 1,6 & 0.9 \\
\hline 67940 & 2014-09-15 & 03:14 & 1.709 & $3 \times 200$ & 1,6 & 0.9 \\
\hline 68114 & 2014-09-17 & $01: 22$ & 1.142 & $3 \times 250$ & 6 & 1.0 \\
\hline 68685 & 2014-10-12 & $00: 30$ & 1.406 & $4 \times 250$ & 6 & 0.9 \\
\hline 69266 & 2014-12-18 & 01:52 & 1.218 & $3 \times 250$ & 1 & 3.0 \\
\hline 69706 & 2014-09-15 & $04: 56$ & 1.023 & $3 \times 500$ & 1,6 & 0.8 \\
\hline 70312 & 2014-09-18 & 03:01 & 1.088 & $3 \times 300$ & 1,4 & 1.3 \\
\hline 70361 & $2014-12-17$ & 03:38 & 1.180 & $3 \times 200$ & 2 & 1.7 \\
\hline 70427 & 2015-01-14 & 05:06 & 1.606 & $3 \times 250$ & 1,3 & 1.7 \\
\hline 70511 & $2014-09-15$ & 04:20 & 1.010 & $3 \times 300$ & 1,6 & 0.7 \\
\hline 71932 & $2014-10-13$ & $02: 22$ & 1.176 & $3 \times 200$ & 1,2 & 1.2 \\
\hline 72047 & $2014-11-25$ & $22: 13$ & 1.027 & $3 \times 350$ & 1 & 1.3 \\
\hline 72143 & 2014-09-19 & 23:39 & 1.414 & $3 \times 250$ & 1,6 & 1.3 \\
\hline 72230 & 2014-09-19 & 01:40 & 1.308 & $2 \times 300$ & 4,6 & 0.9 \\
\hline 72292 & $2014-12-18$ & $00: 57$ & 1.276 & $3 \times 450$ & 1 & 2.0 \\
\hline 72308 & 2014-08-16 & 04:04 & 1.304 & $3 \times 250$ & 5 & 0.7 \\
\hline 72384 & $2014-11-25$ & $21: 49$ & 1.201 & $3 \times 250$ & 1 & 1.4 \\
\hline 72941 & 2014-10-13 & 03:57 & 1.997 & $3 \times 300$ & 1,2 & 1.6 \\
\hline 73860 & 2014-09-14 & $03: 27$ & 1.177 & $3 \times 250$ & 1,6 & 0.9 \\
\hline 74755 & $2014-11-25$ & $22: 53$ & 1.017 & $3 \times 300$ & 1 & 1.2 \\
\hline 74962 & 2014-12-02 & $04: 21$ & 1.136 & $3 \times 500$ & 2 & 1.4 \\
\hline 75089 & 2015-01-19 & $04: 48$ & 1.096 & $3 \times 350$ & 3 & 2.5 \\
\hline 76922 & $2014-10-08$ & 00:02 & 1.292 & $6 \times 250$ & 1,6 & 0.7 \\
\hline 77421 & 2014-10-10 & $05: 44$ & 1.040 & $2 \times 500$ & 1,2 & 0.8 \\
\hline 78069 & 2014-12-02 & $05: 29$ & 1.377 & $3 \times 500$ & 2 & 1.7 \\
\hline 78826 & $2014-09-13$ & $04: 53$ & 1.114 & $3 \times 200$ & 1,6 & 0.8 \\
\hline 78889 & $2014-10-12$ & 02:05 & 2.199 & $4 \times 150$ & 6 & 1.4 \\
\hline 79044 & 2014-10-10 & 05:05 & 1.078 & $3 \times 400$ & 1,2 & 0.7 \\
\hline
\end{tabular}

Notes. Check Table 1 for the ID number of each solar analog star. 
A\&A 586, A129 (2016)

Table C.1. continued.

\begin{tabular}{|c|c|c|c|c|c|c|}
\hline Object & Date & UT start & Airmass & Exposure time (s) & SAs & Seeing $\left({ }^{\prime \prime}\right)$ \\
\hline 85727 & $2015-05-04$ & $23: 47$ & 1.176 & $3 \times 250$ & 3,7 & 0.9 \\
\hline 96405 & 2014-09-17 & $22: 15$ & 1.294 & $5 \times 400$ & 1,4 & 1.3 \\
\hline 96463 & 2014-10-13 & 02:48 & 1.862 & $3 \times 400$ & 1,2 & 1.2 \\
\hline 96768 & 2014-10-07 & $23: 23$ & 1.315 & $3 \times 300$ & 1,6 & 0.8 \\
\hline 98345 & 2014-09-17 & 21:39 & 1.392 & $3 \times 400$ & 1,4 & 1.3 \\
\hline 100784 & 2014-09-18 & 04:59 & 1.049 & $5 \times 500$ & 1,4 & 2.0 \\
\hline 106794 & 2014-12-16 & $00: 11$ & 1.602 & $3 \times 300$ & 1,2 & 3.0 \\
\hline 107070 & 2014-10-13 & 03:29 & 1.873 & $3 \times 400$ & 1,2 & 1.3 \\
\hline 107742 & 2014-12-17 & $23: 13$ & 1.214 & $3 \times 500$ & 1 & 2.4 \\
\hline 111789 & 2014-09-20 & 01:19 & 1.283 & $3 \times 400$ & 1,6 & 1.1 \\
\hline 121096 & 2014-10-07 & $22: 20$ & 1.458 & $3 \times 300$ & 1,6 & 0.7 \\
\hline 129818 & 2014-10-10 & 04:31 & 1.098 & $3 \times 500$ & 1,2 & 0.7 \\
\hline 132056 & 2014-10-12 & $02: 33$ & 1.306 & $3 \times 250$ & 6 & 1.1 \\
\hline 132383 & 2014-10-14 & 04:39 & 1.026 & $3 \times 400$ & 1,2 & 1.0 \\
\hline 133123 & 2014-10-07 & $22: 51$ & 1.329 & $3 \times 400$ & 1,6 & 0.7 \\
\hline 133197 & 2014-10-12 & 01:37 & 1.954 & $3 \times 250$ & 6 & 1.0 \\
\hline 133503 & 2014-10-14 & 03:06 & 1.047 & $3 \times 500$ & 1,2 & 1.4 \\
\hline 137397 & 2014-10-14 & 04:10 & 1.057 & $3 \times 300$ & 1,2 & 1.1 \\
\hline 162795 & 2014-10-12 & 01:06 & 1.613 & $3 \times 300$ & 6 & 1.0 \\
\hline 165536 & 2014-12-18 & 02:17 & 1.235 & $3 \times 250$ & 1 & 0.7 \\
\hline 166264 & 2014-09-18 & $23: 28$ & 1.178 & $3 \times 400$ & 4,6 & 1.0 \\
\hline 169066 & 2014-12-16 & $00: 42$ & 1.301 & $3 \times 300$ & 1,2 & 1.8 \\
\hline 170184 & $2014-11-25$ & $23: 23$ & 1.190 & $3 \times 400$ & 1 & 1.7 \\
\hline 174594 & 2014-12-15 & $22: 55$ & 1.049 & $3 \times 250$ & 1,2 & 1.6 \\
\hline 175811 & 2014-12-14 & $02: 57$ & 1.181 & $3 \times 300$ & 2 & 2.0 \\
\hline 177258 & 2014-12-07 & 06:31 & 1.197 & $3 \times 300$ & 2 & 0.9 \\
\hline 178844 & 2014-12-16 & 04:41 & 1.210 & $3 \times 350$ & 1,2 & 1.6 \\
\hline 186446 & 2015-01-21 & 07:01 & 1.163 & $2 \times 600$ & 3 & 1.8 \\
\hline 186714 & 2014-12-14 & 05:32 & 1.574 & $2 \times 350$ & 2 & 2.3 \\
\hline 208048 & $2015-01-20$ & $00: 28$ & 1.224 & $3 \times 600$ & 1,2 & 2.0 \\
\hline 210564 & 2015-01-19 & $23: 35$ & 1.515 & $3 \times 500$ & 1,2 & 2.5 \\
\hline 213825 & 2014-12-02 & $03: 35$ & 1.158 & $3 \times 300$ & 2 & 1.4 \\
\hline 232922 & 2014-09-18 & 23:01 & 1.177 & $3 \times 200$ & 4,6 & 1.0 \\
\hline 242324 & 2015-01-16 & 23:07 & 1.212 & $3 \times 500$ & 2 & 2.0 \\
\hline 243648 & 2014-12-16 & 04:06 & 1.193 & $3 \times 500$ & 1,2 & 1.6 \\
\hline 250431 & 2015-01-19 & $22: 48$ & 1.259 & $3 \times 600$ & 1,2 & 3.0 \\
\hline 251796 & $2015-01-26$ & $04: 20$ & 1.041 & $3 \times 600$ & 3 & 1.5 \\
\hline 252953 & $2015-01-26$ & 03:16 & 1.012 & $3 \times 450$ & 3 & 1.5 \\
\hline 253538 & 2015-01-19 & 22:02 & 1.304 & $3 \times 500$ & 1,2 & 2.5 \\
\hline 253798 & 2015-01-26 & 03:47 & 1.031 & $3 \times 400$ & 3 & 1.8 \\
\hline 256789 & 2014-12-14 & 03:27 & 1.127 & $3 \times 400$ & 2 & 2.0 \\
\hline 262102 & $2015-01-26$ & 05:37 & 1.111 & $3 \times 600$ & 3 & 1.6 \\
\hline 265259 & $2015-01-26$ & 04:59 & 1.109 & $3 \times 600$ & 3 & 1.5 \\
\hline
\end{tabular}


D. Morate et al.: Visible spectroscopy of Erigone collisional family

Table C.2. Physical parameters of the targets.

\begin{tabular}{|c|c|c|c|c|c|c|c|c|c|}
\hline Object & $a(\mathrm{AU})$ & $e$ & $i\left(^{\circ}\right)$ & $H_{V}$ & $D(\mathrm{~km})$ & $\operatorname{err}_{D}$ & $p_{V}$ & $\operatorname{err}_{p V}$ & Class \\
\hline 163 & 2.367 & 0.21 & 4.801 & 9.5 & 81.58 & 3.06 & 0.033 & 0.004 & $\mathrm{Ch}$ \\
\hline 571 & 2.41 & 0.213 & 5.222 & 11.6 & 11.70 & 0.09 & 0.298 & 0.049 & $\mathrm{~S}$ \\
\hline 10992 & 2.363 & 0.205 & 5.022 & 15.1 & 5.48 & 0.28 & 0.062 & 0.014 & $\mathrm{Cgh}$ \\
\hline 11856 & 2.384 & 0.201 & 4.735 & 15.2 & 4.20 & 0.44 & 0.093 & 0.029 & X \\
\hline 18759 & 2.338 & 0.205 & 4.641 & 14.6 & 2.90 & 0.13 & 0.303 & 0.048 & $\mathrm{Sr}$ \\
\hline 19415 & 2.382 & 0.209 & 5.055 & 15.0 & 5.76 & 0.18 & 0.058 & 0.005 & C \\
\hline 20992 & 2.337 & 0.199 & 5.156 & 15.3 & 4.02 & 0.38 & 0.074 & 0.035 & $\mathrm{Xk}$ \\
\hline 23397 & 2.357 & 0.218 & 5.692 & 15.1 & 7.75 & 3.34 & 0.027 & 0.021 & Ch \\
\hline 24037 & 2.378 & 0.198 & 5.568 & 14.7 & 3.87 & 0.58 & 0.129 & 0.031 & $\mathrm{~L}$ \\
\hline 25381 & 2.395 & 0.202 & 4.751 & 16.4 & 4.33 & 0.17 & 0.028 & 0.005 & X \\
\hline 37437 & 2.349 & 0.215 & 5.06 & 15.4 & 5.30 & 0.78 & 0.043 & 0.026 & $\mathrm{Xc}$ \\
\hline 38106 & 2.331 & 0.213 & 4.781 & 15.4 & - & - & - & - & $\mathrm{L}$ \\
\hline 38173 & 2.355 & 0.212 & 4.736 & 15.7 & 3.56 & 0.24 & 0.080 & 0.016 & X \\
\hline 38661 & 2.309 & 0.203 & 4.834 & 15.5 & - & - & - & - & $\mathrm{Sr}$ \\
\hline 39694 & 2.358 & 0.208 & 5.369 & 16.0 & 3.97 & 1.20 & 0.041 & 0.035 & B \\
\hline 39895 & 2.393 & 0.198 & 5.526 & 15.4 & - & - & - & - & S \\
\hline 42155 & 2.38 & 0.203 & 5.225 & 15.1 & 5.93 & 0.93 & 0.049 & 0.018 & Cgh \\
\hline 42552 & 2.356 & 0.208 & 4.938 & 15.2 & 4.90 & 0.33 & 0.051 & 0.014 & $\mathrm{Cgh}$ \\
\hline 44766 & 2.363 & 0.21 & 5.207 & 14.9 & 6.46 & 0.76 & 0.041 & 0.022 & $\mathrm{Cgh}$ \\
\hline 44942 & 2.383 & 0.204 & 5.215 & 15.2 & 4.83 & 0.67 & 0.063 & 0.051 & X \\
\hline 45357 & 2.374 & 0.215 & 5.211 & 15.5 & 3.96 & 0.06 & 0.078 & 0.006 & Cgh \\
\hline 49731 & 2.379 & 0.202 & 5.25 & 15.1 & 5.22 & 1.10 & 0.065 & 0.066 & X \\
\hline 49859 & 2.361 & 0.212 & 5.003 & 14.4 & 7.45 & 2.56 & 0.055 & 0.069 & Cgh \\
\hline 50068 & 2.347 & 0.206 & 5.035 & 14.9 & - & - & - & - & $\mathrm{L}$ \\
\hline 52870 & 2.359 & 0.205 & 4.938 & 15.2 & 5.53 & 0.05 & 0.053 & 0.009 & $\mathrm{~T}$ \\
\hline 52891 & 2.354 & 0.201 & 4.86 & 15.4 & 4.67 & 0.40 & 0.056 & 0.009 & B \\
\hline 56349 & 2.352 & 0.209 & 5.314 & 15.2 & - & - & - & - & B \\
\hline 65354 & 2.351 & 0.208 & 4.799 & 16.0 & 4.13 & 1.59 & 0.034 & 0.020 & $\mathrm{Xc}$ \\
\hline 66309 & 2.353 & 0.208 & 4.873 & 15.3 & 3.45 & 0.46 & 0.094 & 0.023 & $\mathrm{~L}$ \\
\hline 66325 & 2.352 & 0.205 & 5.125 & 15.4 & 5.63 & 1.87 & 0.042 & 0.088 & Xc \\
\hline 66403 & 2.387 & 0.202 & 5.004 & 15.4 & - & - & - & - & $\mathrm{Ch}$ \\
\hline 67891 & 2.373 & 0.205 & 5.273 & 15.1 & 5.10 & 0.18 & 0.062 & 0.010 & $\mathrm{Ch}$ \\
\hline 67918 & 2.376 & 0.203 & 4.942 & 15.1 & 4.76 & 1.27 & 0.076 & 0.069 & $\mathrm{Ch}$ \\
\hline 67940 & 2.372 & 0.205 & 4.611 & 15.2 & 5.60 & 0.26 & 0.051 & 0.009 & $\mathrm{Ch}$ \\
\hline 68114 & 2.36 & 0.209 & 4.635 & 15.5 & 5.96 & 1.15 & 0.031 & 0.016 & $\mathrm{Cgh}$ \\
\hline 68685 & 2.358 & 0.218 & 5.458 & 15.5 & 4.66 & 0.13 & 0.056 & 0.011 & $\mathrm{Xc}$ \\
\hline 69266 & 2.381 & 0.212 & 5.036 & 15.2 & 5.58 & 1.10 & 0.049 & 0.016 & $X$ \\
\hline 69706 & 2.343 & 0.2 & 4.808 & 15.3 & - & - & - & - & S \\
\hline 70312 & 2.391 & 0.214 & 4.737 & 15.4 & 4.88 & 0.22 & 0.044 & 0.017 & B \\
\hline 70361 & 2.357 & 0.206 & 4.453 & 15.0 & 6.49 & 1.22 & 0.043 & 0.018 & Ch \\
\hline 70427 & 2.37 & 0.208 & 5.087 & 15.1 & 5.56 & 0.01 & 0.051 & 0.001 & X \\
\hline 70511 & 2.402 & 0.203 & 5.165 & 15.2 & - & - & - & - & V \\
\hline 71932 & 2.351 & 0.209 & 4.968 & 16.0 & 4.39 & 0.22 & 0.044 & 0.005 & Cgh \\
\hline 72047 & 2.352 & 0.204 & 4.807 & 15.7 & 4.37 & 1.23 & 0.031 & 0.012 & $\mathrm{Ch}$ \\
\hline 72143 & 2.354 & 0.209 & 5.232 & 15.8 & 4.71 & 0.12 & 0.046 & 0.006 & $\mathrm{Cb}$ \\
\hline 72230 & 2.392 & 0.205 & 4.753 & 15.5 & 4.72 & 0.26 & 0.042 & 0.008 & B \\
\hline 72292 & 2.358 & 0.212 & 5.534 & 15.9 & 4.57 & 0.86 & 0.025 & 0.014 & $\mathrm{Xk}$ \\
\hline 72308 & 2.383 & 0.209 & 4.736 & 15.6 & 5.07 & 1.20 & 0.055 & 0.023 & $\mathrm{Ch}$ \\
\hline 72384 & 2.381 & 0.209 & 5.281 & 15.5 & 3.95 & 0.64 & 0.088 & 0.049 & Ch \\
\hline 72941 & 2.352 & 0.206 & 5.209 & 15.6 & 4.08 & 0.43 & 0.063 & 0.032 & X \\
\hline 73860 & 2.378 & 0.209 & 5.093 & 16.1 & 4.32 & 1.09 & 0.042 & 0.026 & Cgh \\
\hline 74755 & 2.39 & 0.201 & 5.303 & 15.7 & 3.92 & 0.03 & 0.072 & 0.006 & $\mathrm{Cgh}$ \\
\hline 74962 & 2.359 & 0.213 & 5.034 & 15.9 & 4.36 & 0.32 & 0.041 & 0.015 & $\mathrm{Ch}$ \\
\hline 75089 & 2.361 & 0.212 & 5.07 & 15.6 & 4.62 & 0.13 & 0.048 & 0.006 & Cgh \\
\hline 76922 & 2.388 & 0.202 & 5.182 & 15.4 & - & - & - & - & $\mathrm{Xk}$ \\
\hline 77421 & 2.374 & 0.206 & 4.815 & 15.6 & 4.52 & 0.09 & 0.041 & 0.004 & $\mathrm{Xk}$ \\
\hline 78069 & 2.388 & 0.201 & 4.788 & 15.8 & 3.96 & 0.09 & 0.059 & 0.010 & Xk \\
\hline 78826 & 2.356 & 0.217 & 5.52 & 15.7 & 4.93 & 0.97 & 0.042 & 0.032 & $\mathrm{Xc}$ \\
\hline 78889 & 2.388 & 0.218 & 5.055 & 15.8 & 3.76 & 0.51 & 0.072 & 0.022 & $\mathrm{~T}$ \\
\hline 79044 & 2.386 & 0.209 & 4.739 & 16.0 & 3.77 & 0.52 & 0.063 & 0.020 & X \\
\hline 85727 & 2.336 & 0.211 & 5.628 & 15.7 & - & - & - & - & S \\
\hline 96405 & 2.342 & 0.214 & 5.347 & 15.4 & 5.11 & 0.35 & 0.056 & 0.018 & B \\
\hline 96463 & 2.386 & 0.211 & 5.173 & 15.6 & 5.14 & 0.97 & 0.038 & 0.016 & $\mathrm{Cb}$ \\
\hline 96768 & 2.377 & 0.206 & 4.686 & 15.5 & 4.57 & 0.85 & 0.059 & 0.025 & Cgh \\
\hline 98345 & 2.361 & 0.211 & 5.137 & 15.4 & 4.61 & 0.25 & 0.063 & 0.015 & $\mathrm{Cg}$ \\
\hline 100784 & 2.367 & 0.207 & 4.802 & 15.6 & 4.64 & 0.22 & 0.050 & 0.011 & B \\
\hline 106794 & 2.339 & 0.215 & 5.317 & 15.8 & 3.82 & 0.61 & 0.070 & 0.078 & $\mathrm{~T}$ \\
\hline
\end{tabular}


A\&A 586, A129 (2016)

Table C.2. continued.

\begin{tabular}{|c|c|c|c|c|c|c|c|c|c|}
\hline Object & $\bar{a} a(\mathrm{AU})$ & $e$ & $\overline{i\left(^{\circ}\right)}$ & $\overline{H_{V}}$ & $\overline{D(\mathrm{~km})}$ & $\operatorname{err}_{D}$ & $p_{V}$ & $\operatorname{err}_{p V}$ & Class \\
\hline 107070 & 2.406 & 0.177 & 5.419 & 15.9 & - & - & - & - & $\mathrm{L}$ \\
\hline 107742 & 2.399 & 0.199 & 5.48 & 16.1 & 3.39 & 0.64 & 0.067 & 0.022 & B \\
\hline 111789 & 2.383 & 0.203 & 5.208 & 15.7 & 4.01 & 0.09 & 0.069 & 0.015 & $\mathrm{Ch}$ \\
\hline 121096 & 2.346 & 0.211 & 5.338 & 15.8 & 4.10 & 0.15 & 0.036 & 0.005 & X \\
\hline 129818 & 2.347 & 0.208 & 4.854 & 16.0 & 3.66 & 0.64 & 0.069 & 0.027 & $\mathrm{Cb}$ \\
\hline 132056 & 2.35 & 0.205 & 5.17 & 15.9 & 3.96 & 0.47 & 0.053 & 0.015 & $\mathrm{Ch}$ \\
\hline 132383 & 2.357 & 0.21 & 5.149 & 15.6 & 1.77 & 0.42 & 0.356 & 0.156 & $\mathrm{~L}$ \\
\hline 133123 & 2.361 & 0.214 & 5.261 & 16.2 & 3.24 & 0.04 & 0.062 & 0.009 & B \\
\hline 133197 & 2.386 & 0.209 & 5.078 & 15.8 & 3.96 & 0.09 & 0.065 & 0.015 & $\mathrm{~T}$ \\
\hline 133503 & 2.349 & 0.211 & 5.042 & 16.3 & 3.02 & 0.02 & 0.058 & 0.008 & $\mathrm{Cgh}$ \\
\hline 137397 & 2.393 & 0.202 & 4.972 & 16.3 & 2.83 & 0.43 & 0.067 & 0.027 & $\mathrm{Ch}$ \\
\hline 162795 & 2.361 & 0.212 & 5.388 & 16.2 & 3.83 & 0.19 & 0.048 & 0.014 & $\mathrm{~T}$ \\
\hline 165536 & 2.354 & 0.21 & 5.064 & 16.0 & 3.58 & 0.65 & 0.057 & 0.030 & $\mathrm{Cgh}$ \\
\hline 166264 & 2.381 & 0.177 & 5.199 & 15.9 & - & - & - & - & $\mathrm{Xk}$ \\
\hline 169066 & 2.386 & 0.212 & 5.316 & 15.9 & 4.93 & 0.25 & 0.038 & 0.005 & X \\
\hline 170184 & 2.347 & 0.208 & 5.234 & 16.3 & 3.69 & 0.85 & 0.039 & 0.014 & Xk \\
\hline 174594 & 2.353 & 0.215 & 5.427 & 16.3 & 2.84 & 0.65 & 0.096 & 0.040 & $\mathrm{Ch}$ \\
\hline 175811 & 2.379 & 0.214 & 4.802 & 15.7 & 3.91 & 0.57 & 0.057 & 0.027 & $\mathrm{Cgh}$ \\
\hline 177258 & 2.325 & 0.214 & 5.162 & 16.9 & 2.49 & 0.57 & 0.050 & 0.028 & B \\
\hline 178844 & 2.373 & 0.206 & 5.123 & 16.2 & 3.61 & 0.43 & 0.045 & 0.008 & $\mathrm{Cgh}$ \\
\hline 186446 & 2.378 & 0.199 & 5.117 & 16.0 & - & - & - & - & $\mathrm{Ch}$ \\
\hline 186714 & 2.404 & 0.215 & 5.207 & 16.9 & 2.33 & 0.53 & 0.062 & 0.028 & $\mathrm{Cgh}$ \\
\hline 208048 & 2.389 & 0.213 & 4.844 & 16.5 & 3.26 & 0.38 & 0.052 & 0.013 & $\mathrm{~T}$ \\
\hline 210564 & 2.414 & 0.21 & 5.33 & 16.6 & 3.04 & 0.35 & 0.046 & 0.018 & X \\
\hline 213825 & 2.356 & 0.207 & 4.882 & 16.1 & 3.40 & 0.60 & 0.061 & 0.021 & Cgh \\
\hline 232922 & 2.348 & 0.215 & 5.17 & 16.0 & 3.78 & 0.15 & 0.054 & 0.009 & $\mathrm{Ch}$ \\
\hline 242324 & 2.411 & 0.205 & 4.952 & 17.0 & 2.49 & 0.70 & 0.059 & 0.048 & $\mathrm{Ch}$ \\
\hline 243648 & 2.387 & 0.193 & 5.093 & 16.9 & 2.56 & 0.05 & 0.052 & 0.008 & X \\
\hline 250431 & 2.398 & 0.211 & 5.337 & 16.7 & 3.29 & 0.59 & 0.037 & 0.013 & $\mathrm{~T}$ \\
\hline 251796 & 2.341 & 0.204 & 4.826 & 16.8 & 3.08 & 0.91 & 0.035 & 0.022 & $X$ \\
\hline 252953 & 2.401 & 0.202 & 4.749 & 16.3 & 3.58 & 1.04 & 0.046 & 0.040 & $\mathrm{Ch}$ \\
\hline 253538 & 2.393 & 0.217 & 5.526 & 16.4 & 3.17 & 0.91 & 0.048 & 0.044 & B \\
\hline 253798 & 2.332 & 0.201 & 4.732 & 16.8 & 2.60 & 0.43 & 0.055 & 0.020 & $\mathrm{Ch}$ \\
\hline 256789 & 2.373 & 0.207 & 4.857 & 16.8 & 2.54 & 0.32 & 0.052 & 0.010 & X \\
\hline 262102 & 2.355 & 0.207 & 4.891 & 17.2 & 3.01 & 0.79 & 0.026 & 0.025 & $\mathrm{Ch}$ \\
\hline 265259 & 2.404 & 0.21 & 4.981 & 16.9 & 3.18 & 0.58 & 0.036 & 0.015 & $X$ \\
\hline
\end{tabular}


D. Morate et al.: Visible spectroscopy of Erigone collisional family

Table C.3. Summary of the obtained results for the primitive asteroids in our sample.

\begin{tabular}{|c|c|c|c|c|c|}
\hline Object & Class & Slope $(\% / 1000 \AA)$ & Band & $\lambda_{\text {cent }}(\AA)$ & Depth (\%) \\
\hline 163 & $\mathrm{Ch}$ & $1.34 \pm 0.61$ & YES & $6899 \pm 26$ & $1.52 \pm 0.07$ \\
\hline 10992 & $\mathrm{Cgh}$ & $0.95 \pm 0.63$ & YES & $7074 \pm 37$ & $1.80 \pm 0.14$ \\
\hline 11856 & $\mathrm{X}$ & $4.94 \pm 0.71$ & NO & - & - \\
\hline 19415 & $\mathrm{C}$ & $-0.56 \pm 0.66$ & NO & - & - \\
\hline 20992 & $\mathrm{Xk}$ & $-0.17 \pm 0.64$ & NO & - & - \\
\hline 23397 & $\mathrm{Ch}$ & $1.95 \pm 0.65$ & YES & $6957 \pm 78$ & $4.43 \pm 0.22$ \\
\hline 25381 & $\mathrm{X}$ & $2.16 \pm 0.63$ & NO & - & - \\
\hline 37437 & $\mathrm{Xc}$ & $3.65 \pm 0.76$ & NO & - & - \\
\hline 38173 & $X$ & $4.90 \pm 0.70$ & YES & $7149 \pm 60$ & $4.32 \pm 0.28$ \\
\hline 39694 & B & $-0.77 \pm 0.65$ & YES & $6887 \pm 98$ & $2.23 \pm 0.30$ \\
\hline 42155 & $\mathrm{Cgh}$ & $1.07 \pm 0.61$ & YES & $6961 \pm 35$ & $1.34 \pm 0.08$ \\
\hline 42552 & $\mathrm{Cgh}$ & $1.33 \pm 0.62$ & YES & $7107 \pm 33$ & $2.26 \pm 0.10$ \\
\hline 44766 & $\mathrm{Cgh}$ & $1.29 \pm 0.61$ & YES & $7054 \pm 33$ & $1.48 \pm 0.08$ \\
\hline 44942 & $\mathrm{X}$ & $4.10 \pm 0.63$ & YES & $7089 \pm 81$ & $2.69 \pm 0.16$ \\
\hline 45357 & $\mathrm{Cgh}$ & $1.75 \pm 0.62$ & YES & $6913 \pm 68$ & $2.76 \pm 0.11$ \\
\hline 49731 & $\mathrm{X}$ & $2.85 \pm 0.62$ & NO & - & - \\
\hline 49859 & $\mathrm{Cgh}$ & $2.15 \pm 0.61$ & YES & $6936 \pm 32$ & $1.83 \pm 0.08$ \\
\hline 52870 & $\mathrm{~T}$ & $5.99 \pm 0.77$ & NO & - & - \\
\hline 52891 & B & $-2.13 \pm 0.64$ & NO & - & - \\
\hline 56349 & B & $-0.85 \pm 0.87$ & $\mathrm{NO}$ & - & - \\
\hline 65354 & $\mathrm{Xc}$ & $1.78 \pm 0.66$ & NO & - & - \\
\hline 66325 & $\mathrm{Xc}$ & $3.13 \pm 0.62$ & NO & - & - \\
\hline 66403 & $\mathrm{Ch}$ & $1.87 \pm 0.61$ & YES & $7023 \pm 30$ & $2.00 \pm 0.07$ \\
\hline 67891 & $\mathrm{Ch}$ & $1.83 \pm 0.63$ & YES & $7348 \pm 75$ & $1.75 \pm 0.20$ \\
\hline 67918 & $\mathrm{Ch}$ & $0.05 \pm 0.61$ & YES & $7052 \pm 32$ & $2.20 \pm 0.08$ \\
\hline 67940 & $\mathrm{Ch}$ & $1.11 \pm 0.62$ & YES & $7149 \pm 53$ & $1.25 \pm 0.11$ \\
\hline 68114 & $\mathrm{Cgh}$ & $1.63 \pm 0.61$ & YES & $7153 \pm 33$ & $1.29 \pm 0.07$ \\
\hline 68685 & $\mathrm{Xc}$ & $2.18 \pm 0.74$ & NO & - & - \\
\hline 69266 & $\mathrm{X}$ & $3.07 \pm 0.61$ & NO & - & - \\
\hline 70312 & B & $-0.74 \pm 0.62$ & YES & $6996 \pm 35$ & $2.00 \pm 0.10$ \\
\hline 70361 & $\mathrm{Ch}$ & $0.63 \pm 0.61$ & YES & $7049 \pm 32$ & $1.96 \pm 0.08$ \\
\hline 70427 & $\mathrm{X}$ & $2.65 \pm 0.61$ & YES & $6890 \pm 68$ & $1.39 \pm 0.14$ \\
\hline 71932 & $\mathrm{Cgh}$ & $1.18 \pm 0.62$ & YES & $7199 \pm 48$ & $1.74 \pm 0.12$ \\
\hline 72047 & $\mathrm{Ch}$ & $1.16 \pm 0.62$ & YES & $7125 \pm 52$ & $1.79 \pm 0.16$ \\
\hline 72143 & $\mathrm{Cb}$ & $0.96 \pm 0.68$ & NO & - & - \\
\hline 72230 & B & $-1.20 \pm 0.78$ & NO & - & - \\
\hline 72292 & $\mathrm{Xk}$ & $4.63 \pm 0.74$ & NO & - & - \\
\hline 72308 & $\mathrm{Ch}$ & $1.34 \pm 0.61$ & YES & $7050 \pm 32$ & $1.76 \pm 0.06$ \\
\hline 72384 & $\mathrm{Ch}$ & $1.63 \pm 0.61$ & YES & $7018 \pm 33$ & $1.64 \pm 0.07$ \\
\hline 72941 & $\mathrm{X}$ & $2.92 \pm 0.66$ & YES & $7380 \pm 102$ & $3.24 \pm 0.20$ \\
\hline 73860 & $\mathrm{Cgh}$ & $1.81 \pm 0.61$ & YES & $7230 \pm 65$ & $2.49 \pm 0.12$ \\
\hline 74755 & $\mathrm{Cgh}$ & $0.71 \pm 0.62$ & YES & $7118 \pm 50$ & $2.34 \pm 0.16$ \\
\hline 74962 & $\mathrm{Ch}$ & $1.50 \pm 0.67$ & YES & $6922 \pm 47$ & $4.02 \pm 0.23$ \\
\hline 75089 & $\mathrm{Cgh}$ & $1.65 \pm 0.68$ & YES & $7200 \pm 108$ & $4.15 \pm 0.37$ \\
\hline 76922 & $\mathrm{Xk}$ & $5.06 \pm 0.78$ & NO & - & - \\
\hline 77421 & $\mathrm{Xk}$ & $1.78 \pm 0.65$ & NO & - & - \\
\hline 78069 & $\mathrm{Xk}$ & $2.51 \pm 0.76$ & NO & - & - \\
\hline 78826 & $\mathrm{Xc}$ & $2.22 \pm 0.61$ & $\mathrm{NO}$ & - & - \\
\hline 78889 & $\mathrm{~T}$ & $3.62 \pm 0.66$ & NO & - & - \\
\hline 79044 & $\mathrm{X}$ & $1.76 \pm 0.63$ & NO & - & - \\
\hline 96405 & B & $-2.02 \pm 0.63$ & NO & - & - \\
\hline 96463 & $\mathrm{Cb}$ & $2.49 \pm 0.65$ & NO & - & - \\
\hline 96768 & $\mathrm{Cgh}$ & $0.23 \pm 0.62$ & YES & $6642 \pm 41$ & $3.41 \pm 0.19$ \\
\hline 98345 & $\mathrm{Cg}$ & $-0.72 \pm 0.86$ & NO & - & - \\
\hline 100784 & $\mathrm{~B}$ & $-2.01 \pm 0.83$ & NO & - & - \\
\hline 106794 & $\mathrm{~T}$ & $5.55 \pm 0.80$ & YES & $7364 \pm 202$ & $3.52 \pm 0.45$ \\
\hline 107742 & B & $-1.74 \pm 0.80$ & NO & - & - \\
\hline 111789 & $\mathrm{Ch}$ & $2.24 \pm 0.64$ & YES & $7073 \pm 32$ & $3.32 \pm 0.11$ \\
\hline 121096 & $X$ & $2.76 \pm 0.69$ & YES & $6853 \pm 250$ & $2.23 \pm 0.33$ \\
\hline 129818 & $\mathrm{Cb}$ & $1.45 \pm 1.08$ & NO & - & - \\
\hline 132056 & $\mathrm{Ch}$ & $0.48 \pm 0.65$ & YES & $7108 \pm 63$ & $4.20 \pm 0.24$ \\
\hline 133123 & B & $-0.58 \pm 0.71$ & YES & $7282 \pm 137$ & $4.28 \pm 0.36$ \\
\hline 133197 & $\mathrm{~T}$ & $3.92 \pm 0.67$ & NO & - & - \\
\hline 133503 & $\mathrm{Cgh}$ & $1.15 \pm 0.63$ & YES & $6898 \pm 76$ & $2.83 \pm 0.27$ \\
\hline 137397 & $\mathrm{Ch}$ & $0.92 \pm 0.62$ & YES & $6740 \pm 46$ & $2.67 \pm 0.14$ \\
\hline 162795 & $\mathrm{~T}$ & $2.93 \pm 0.65$ & NO & - & - \\
\hline 165536 & $\mathrm{Cgh}$ & $2.38 \pm 0.61$ & YES & $7102 \pm 53$ & $1.44 \pm 0.10$ \\
\hline
\end{tabular}


Table C.3. continued.

\begin{tabular}{cccccc}
\hline \hline Object & Class & Slope $(\% / 1000 \AA)$ & Band & $\lambda_{\text {cent }}(\AA)$ & Depth $(\%)$ \\
\hline 166264 & Xk & $2.86 \pm 0.78$ & NO & - & - \\
169066 & X & $4.50 \pm 0.70$ & YES & $6974 \pm 59$ & $3.08 \pm 0.32$ \\
170184 & Xk & $1.11 \pm 0.72$ & NO & - & - \\
174594 & Ch & $2.41 \pm 0.64$ & YES & $7051 \pm 46$ & $2.09 \pm 0.16$ \\
175811 & Cgh & $0.61 \pm 0.61$ & YES & $7093 \pm 31$ & $2.36 \pm 0.07$ \\
177258 & B & $-1.41 \pm 0.81$ & NO & - & - \\
178844 & Cgh & $1.29 \pm 0.62$ & YES & $7058 \pm 42$ & $2.52 \pm 0.13$ \\
186446 & Ch & $1.10 \pm 1.08$ & YES & $7081 \pm 26$ & $6.06 \pm 0.74$ \\
186714 & Cgh & $3.25 \pm 0.68$ & YES & $7145 \pm 66$ & $5.50 \pm 0.29$ \\
208048 & T & $5.74 \pm 0.64$ & NO & - & - \\
210564 & X & $7.22 \pm 0.78$ & YES & $7009 \pm 24$ & $9.14 \pm 0.58$ \\
213825 & Cgh & $0.93 \pm 0.61$ & YES & $7019 \pm 48$ & $2.42 \pm 0.13$ \\
232922 & Ch & $-0.23 \pm 0.61$ & YES & $7109 \pm 28$ & $1.50 \pm 0.06$ \\
242324 & Ch & $2.30 \pm 0.64$ & YES & $7420 \pm 87$ & $2.81 \pm 0.29$ \\
243648 & X & $6.20 \pm 0.62$ & YES & $6957 \pm 93$ & $1.85 \pm 0.17$ \\
250431 & T & $6.59 \pm 0.97$ & NO & - & - \\
251796 & X & $1.43 \pm 0.68$ & NO & - & - \\
252953 & Ch & $1.17 \pm 0.64$ & YES & $7127 \pm 87$ & $4.60 \pm 0.21$ \\
253538 & B & $-2.79 \pm 0.92$ & YES & $7199 \pm 118$ & $6.49 \pm 0.78$ \\
253798 & Ch & $0.65 \pm 0.62$ & YES & $6755 \pm 42$ & $2.42 \pm 0.12$ \\
256789 & X & $4.01 \pm 0.65$ & NO & - & - \\
262102 & Ch & $2.85 \pm 0.70$ & YES & $6816 \pm 134$ & $4.21 \pm 0.28$ \\
265259 & $\mathrm{X}$ & $2.80 \pm 0.71$ & NO & - & - \\
\hline & & & & &
\end{tabular}

